ISSN 2227-7099

www.mdpi.com/journal/economies

\title{
Article
}

\section{Measuring Voting Power in Convex Policy Spaces}

\section{Sascha Kurz}

Department of Mathematics, University of Bayreuth, Universitätsstr. 30, Bayreuth D-95440, Germany; E-Mail: sascha.kurz@uni-bayreuth.de; Tel.: +49-921-55-7353; Fax: +49-921-55-7352

Received: 20 December 2013; in revised form: 6 February 2014 / Accepted: 20 February 2014 / Published: 6 March 2014

\begin{abstract}
Classical power index analysis considers the individual's ability to influence the aggregated group decision by changing its own vote, where all decisions and votes are assumed to be binary. In many practical applications we have more options than either "yes" or "no". Here we generalize three important power indices to continuous convex policy spaces. This allows the analysis of a collection of economic problems like, e.g., tax rates or spending that otherwise would not be covered in binary models.
\end{abstract}

Keywords: power; single peaked preferences; convex policy space; group decision making; Shapley-Shubik index; Banzhaf index; nucleolus; simple games; multiple levels of approval

\section{Introduction}

Important decisions are likely made by groups of experts or in the democratic decision-making context by voters. Giving a set of experts opinions or the votes of the population or representatives within a committee, usually the aggregated decision is (deterministically) determined according to a certain decision rule. Examples of such decision (or aggregation) rules are the majority rule, which selects the alternative that has a majority, or more generally weighted voting rules like, e.g., those used by the US Electoral College or the EU Council of Ministers.

Given such a decision rule it is quite natural to ask for the individual power, by which we understand the ability to influence the aggregated decision, of the committee members or voters. A lot of literature is concerned with measuring this voting power under certain circumstances. However, in our opinion the answers given so far are not completely satisfactory. To this end we quote [1]:

"Scientists who study power in political and economic institutions seem divided into two disjoint methodological camps. The first one uses non-cooperative game theory 
to analyze the impact of explicit decision procedures and given preferences over a well-defined-usually Euclidean-policy space. The second one stands in the tradition of cooperative game theory with much more abstractly defined voting bodies: the considered agents have no particular preferences and form winning coalitions which implement unspecified policies. Individual chances of being part of and influencing a winning coalition are then measured by a power index.

Several authors have concluded that it is time to develop a unified framework for measuring decision power (cf. [2,3])."

A similar opinion is, e.g., shared in [4,5]. Within the tradition of [1] we try to develop such a unified framework for measuring decision power. Our starting point is the well developed theory of so-called simple games, see [6], within the field of cooperative game theory. The big drawback of simple games is that both - the voters and the aggregated decision—are binary. In [7] the authors propose abstention as a third option for the voters, which they argue to occur quite frequently in practice. At the end of their paper they drastically conclude ignoring abstention causes serious errors when evaluating the power of real-world voting systems:

"It seems that we are confronted here with a clear-cut case of theory-laden (or theory-biased) observation. Scientists, equipped with a ready-made theoretical conception, "observe" in reality phenomena that fit that conception. And where the phenomena do not quite fit the theory, they are at best consciously ignored, but more often actually misperceived and tweaked into the theoretical mould."

This statement causes several follow-up papers. In [8] the authors considered simple games with multiple levels of approval. If the voters can choose between $j$ (ordered) alternatives and the aggregated decision is taken between $k$ (ordered) alternatives, those games are called $(j, k)$ simple games, so that the examples of [7] fit in as (3,2)-simple games. We remark that other authors consider similar extensions where the alternatives are not ordered, see, e.g., [9-14]. Power indices for $(j, k)$ simple games were, e.g., introduced in $[15,16]$, while the basic ideas have been developed for the special case of $(3,2)$ simple games in earlier papers like, e.g., [7].

In this paper we want to consider convex policy spaces with a continuum of alternatives. To keep things simple and partially supported by empirical evidence, i.e., the authors of [17] show that the individuals (more-dimensional) opinions can often be well approximated by a 1-dimensional line, we assume the policy space to be 1-dimensional. Moreover we normalize the policy space, of both the input and the output, to the real interval $[0,1]$. To be more precisely we consider the real interval $[0,1]$ of policy alternatives with single peaked preferences. In some sense those games can be considered as the limit of $(j, k)$ simple games, where both $j$ and $k$ tend to infinity. Going from binary $\{0,1\}$-decisions to continuous $[0,1]$-decisions allows the analysis of a collection of economic problems like, e.g., tax rates or spending that otherwise would not be covered in binary models.

Almost the whole literature on voting power is limited to binary or discrete models-a few exceptions are given by, e.g., $[1,3,18-21]$. 
The aim of this paper is to propose a generalization of some of the notions for simple games with binary $\{0,1\}$-decisions to continuous $[0,1]$-decisions in a consistent way. To this end we review some basic definitions and results for binary simple games in Section 2. Some of the usual notation is slightly modified so that the coincidence with our definitions for the continuous case becomes more transparent. In Section 3 we briefly introduce $(j, k)$ simple games. Directly after, we propose basic definitions and first results for continuous simple games in Section 4. In Section 5 we give some examples of continuous simple games. Generalized versions of three selected power indices are given in Section 6. The case of vote distributions, where not all alternatives are equiprobable, is briefly treated in Section 7 . We conclude in Section 8 and suggest some research question which may carry forward the development of a unified theory of power measurement. Some power index computations for examples of certain continuous simple games are delayed to an appendix.

\section{Binary Decision Rules}

A binary decision (or aggregation) rule can be modeled as a function $v: 2^{N} \rightarrow\{0,1\}$ mapping the coalition $S$ of supporters, i.e., those who vote "yes", to the aggregated group decision $v(S) \in\{0,1\}$. Within the remaining part of the paper we denote by $N=\{1,2, \ldots, n\}$ the set of $n \in \mathbb{N}$ voters and by $2^{N}$ its power set, i.e., the set of its subsets. In the following we specialize those Boolean functions more and more by requiring desirable properties of a binary decision rule. Quite naturally we require:

(1) if no voter is in favor of a proposal, reject it;

(2) if all voters are in favor of a proposal, accept it.

Definition 1. A Boolean game is a function $v: 2^{N} \rightarrow\{0,1\}$ with $v(\emptyset)=0$ and $v(N)=1$. The set of all Boolean games on $n$ players is denoted by $\mathcal{B}_{n}$.

We remark that some authors drop the condition $v(N)=1$. When the group of supporting voters is enlarged we would usually expect that the group decision does not change from acceptance to rejection. This is formalized in:

Definition 2. A simple game is a Boolean game $v: 2^{N} \rightarrow\{0,1\}$ such that $v(S) \leq v(T)$ for all $\emptyset \subseteq S \subseteq T \subseteq N$. The set of all simple games on n players is denoted by $\mathcal{S}_{n}$.

We call subsets $S \subseteq N$ coalitions. Those coalitions come in two types, i.e., either we have $v(S)=1$ or $v(S)=0$. We speak of a winning coalition in the first and of a losing coalition in the second case. The set of all winning or the set of all losing coalitions are both sufficient to uniquely characterize a simple game. A winning coalition, with the property that all proper subsets are losing, is called minimal winning. Similarly, we call a losing coalition maximal losing if all of its proper supersets are winning.

Definition 3. Let $v$ be a simple game. By $\mathcal{W}$ we denote the set of all winning and by $\mathcal{W}^{m}$ we denote the set of all minimal winning coalitions of $v$. Similarly, by $\mathcal{L}$ we denote the set of all losing and by $\mathcal{L}^{M}$ we denote the set of all maximal losing coalitions of $v$.

We remark that both $\mathcal{W}^{m}$ and $\mathcal{L}^{M}$ are sufficient to uniquely characterize a simple game. An example of a simple game for three players is given by $\mathcal{W}=\{\{1,2\},\{1,2,3\}\}$. The remaining six coalitions in $N \backslash \mathcal{W}$ are losing. The unique minimal winning coalition is given by $\{1,2\}$ and $\mathcal{L}^{M}=\{\{1,3\},\{2,3\}\}$. 
Definition 4. A voter that is not contained in any minimal winning coalition is called a null voter.

In our previous example voter 3 is a null voter. For the next definition we assume that we let our committee decide on a certain proposal and its logical negation. It may be seen as somewhat strange if both proposals would be accepted under the same preferences of the voters. So anticipating the next definition, we can state that the most studied simple games are generally proper.

Definition 5. A simple game is called proper if the complement $N \backslash S$ of any winning coalition $S$ is losing. It is called strong if the complement $N \backslash S$ of any losing coalition $S$ is winning. A simple game that is both proper and strong is called constant-sum (or self-dual, or decisive).

The desirability relation, introduced in [22], assumes a certain intuitive ordering of the voters:

Definition 6. Given a simple game, characterized by its set of winning coalitions $\mathcal{W} \subseteq 2^{N}$, we say that voter $i \in N$ is more desirable as voter $j \in N$, denoted by $i \succeq j$, if

(1) for all $S \subseteq N \backslash\{i, j\}$ with $S \cup\{j\} \in \mathcal{W}$, we have $S \cup\{i\} \in \mathcal{W}$;

(2) for all $S \subseteq N \backslash\{i, j\}$ with $S \cup\{i\} \in \mathcal{L}$, we have $S \cup\{j\} \in \mathcal{L}=2^{N} \backslash \mathcal{W}$.

We write $i \simeq j$ if $i \succeq j, j \succeq i$ and use $i \succ j$ as abbreviation for $i \succeq j, i \not z j$.

As an abbreviation we write $(\mathcal{W}, N)$ for a Boolean game with $\mathcal{W} \subseteq 2^{N}$ as its set of winning coalitions.

Definition 7. A simple game $(\mathcal{W}, N)$ is called complete if for each pair of voters $i, j \in N$ we have $i \succeq j$ or $j \succeq i$. The set of all complete (simple) games on $n$ voters is denoted by $\mathcal{C}_{n}$.

We remark that our previous example of a simple game is complete and we have $1 \simeq 2 \succeq 3$.

Definition 8. Let $(\mathcal{W}, N)$ be a complete simple game, where $1 \succeq 2 \succeq \cdots \succeq n$, and $S \subseteq N$ be arbitrary. A coalition $T \subseteq N$ is a direct left-shift of $S$ whenever there exists a voter $i \in S$ with $i-1 \notin S$ such that $T=S \backslash\{i\} \cup\{i-1\}$ for $i>1$ or $T=S \cup\{n\}$ for $n \notin S$. Similarly, a coalition $T \subseteq N$ is a direct right-shift of $S$ whenever there exists a voter $i \in S$ with $i+1 \notin S$ such that $T=S \backslash\{i\} \cup\{i+1\}$ for $i<n$ or $T=S \backslash\{n\}$ for $n \in S$. A coalition $T$ is a left-shift of $S$ if it arises as a sequence of direct left-shifts. Similarly, it is a right-shift of $S$ if it arises as a sequence of direct right-shifts. A winning coalition $S$ such that all right-shifts of $S$ are losing is called shift-minimal (winning). Similarly, a winning coalition $S$ such that all left-shifts of $S$ are winning is called shift-maximal (losing). By $\mathcal{W}^{\text {sm }}$ we denote the set of all shift-minimal minimal winning coalitions of $(\mathcal{W}, N)$ and by $\mathcal{L}^{s M}$ the set of all shift-maximal losing coalitions.

In our example we have $\mathcal{W}^{\text {sm }}=\{\{1,2\}\}$ and $\mathcal{L}^{s M}=\{\{1,3\}\}$ since $\{2,3\}$ is a direct right-shift of $\{1,3\}$. Both of the sets $\mathcal{W}^{s m}$ or $\mathcal{L}^{s M}$ are sufficient to uniquely characterize a complete simple game. Some simple games permit a more compact representation using just $n+1$ non-negative real numbers:

Definition 9. A simple game $(\mathcal{W}, N)$ is weighted if there exists a quota $q>0$ and weights $w_{1}, \ldots, w_{n} \geq 0$ such that coalition $S$ is winning if and only if $w(S)=\sum_{i \in S} w_{i} \geq q$. We denote the corresponding game by $\left[q ; w_{1}, \ldots, w_{n}\right]$. The set of all weighted (simple) games on $n$ voters is denoted by $\mathcal{T}_{n}$. 
We remark that each weighted game $v$ admits several weighted representations $[q ; w]$, e.g., if $[q ; w]$ is a weighted representation for $v$, then $[\lambda \cdot q ; \lambda \cdot w]$ is also a weighted representation for all $\lambda \in \mathbb{R}_{>0}$. The set of weighted representations of a given weighted game $v$ is even more involved. As an example we remark that $[2 ; 1,1,1]$ and $[5 ; 4,3,2]$ represent the same game. Our initial example of a simple game can be written as $[2 ; 1,1,0]$.

We remark that all weighted simple games are complete. Not every complete simple game is weighted $^{1}$, but every simple game is the intersection of finitely many weighted games. The minimum number needed is called dimension of the simple game. The number $\left|\mathcal{C}_{n}\right|$ of complete simple games with $n$ voters grows much faster than the number $\left|\mathcal{T}_{n}\right|$ of weighted simple games with $n$ voters, see, e.g., [23]. Similarly, the number $\left|\mathcal{S}_{n}\right|$ of simple games with $n$ voters grows much faster than the number $\left|\mathcal{C}_{n}\right|$ of complete simple games with $n$ voters, see, e.g., [24].

In the following we will commonly consider so-called normalized weights $w \in \mathbb{R}_{>0}^{n}$, where $\|w\|_{1}=\sum_{i=1}^{n} w_{i}=1$. As an abbreviation we use $w(S)=\sum_{i \in S} w_{i}$ for coalitions $S \subseteq N$. For weighted simple games the properties proper, strong, and constant-sum are ultimately linked with the quota $q$ :

Lemma 1. A weighted game $v$ with normalized weights $w \in \mathbb{R}_{\geq 0}^{n}$, i.e., $\|w\|_{1}=1$, and quota $q \in(0,1]$ is proper if and only if there exists a weighted representation with normalized weights $w^{\prime} \in \mathbb{R}_{\geq 0}^{n}$ and quota $q^{\prime} \in\left(\frac{1}{2}, 1\right]$.

Proof. If $q>\frac{1}{2}$, then for each winning coalition $S$ with $w(S) \geq q$ we have $w(N \backslash S)=1-w(S) \leq$ $1-q<\frac{1}{2}<q$ so that $N \backslash S$ has to be losing and the game $v$ is proper.

For the other direction we assume that $v$ is proper. From Definition 1 we conclude $w \neq \mathbf{0}$, so that we can set $w^{\prime}=\frac{w}{\|w\|_{1}}$, i.e., we normalize the weights to sum 1 . Next we set

$$
q_{1}=\max _{S \in \mathcal{L}} w^{\prime}(S) \quad \text { and } \quad q_{2}=\max _{S \in \mathcal{W}} w^{\prime}(S)
$$

where obviously $0 \leq q_{1}<q_{2} \leq 1$ due to the definition of a weighted game. Each choice of $q^{\prime} \in\left(q_{1}, q_{2}\right]$ corresponds to the same weighted game. Thus it remains to prove that $q_{2}>\frac{1}{2}$. Assume to the contrary $q_{2} \leq \frac{1}{2}$. Let $S \in \mathcal{W} \neq \emptyset$ be an arbitrary winning coalition. Since

$$
w^{\prime}(N \backslash S)=w^{\prime}(N)-w^{\prime}(S)=1-w^{\prime}(S) \geq 1-q_{2} \geq \frac{1}{2} \geq q_{2}
$$

the complementary coalition $N \backslash S$ would be also winning, which is a contradiction to the assumption that $v$ is proper.

Lemma 2. A weighted game $v$ with normalized weights $w \in \mathbb{R}_{\geq 0}^{n}$ and quota $q \in(0,1]$ is strong if and only if there exists a weighted representation with normalized weights $w^{\prime} \in \mathbb{R}_{\geq 0}^{n}$ and quota $q^{\prime} \in\left(0, \frac{1}{2}\right]$.

PROOF. If $q \leq \frac{1}{2}$, then for each losing coalition $S$ with $w(S)<q$ we have $w(N \backslash S)=1-w(S)>$ $1-q \geq \frac{1}{2} \geq q$ so that $N \backslash S$ has to be winning and the game $v$ is strong.

$1 \quad$ We have $\mathcal{S}_{n} \subseteq \mathcal{C}_{n} \subseteq \mathcal{T}_{n}, \mathcal{S}_{n} \neq \mathcal{C}_{n}$ for $n \geq 4$, and $\mathcal{C}_{n} \neq \mathcal{T}_{n}$ for $n \geq 6$ 
For the other direction we assume that $v$ is strong. From Definition 1 we conclude $w \neq \mathbf{0}$, so that we can set $w^{\prime}=\frac{w}{\|w\|_{1}}$, i.e., we normalize the weights to sum 1 . Next we set

$$
q_{1}=\max _{S \in \mathcal{L}} w^{\prime}(S) \quad \text { and } \quad q_{2}=\max _{S \in \mathcal{W}} w^{\prime}(S)
$$

where obviously $0 \leq q_{1}<q_{2} \leq 1$ due to the definition of a weighted game. Each choice of $q^{\prime} \in\left(q_{1}, q_{2}\right]$ corresponds to the same weighted game. Thus it remains to prove that $q_{1}<\frac{1}{2}$. Assume to the contrary $q_{1} \geq \frac{1}{2}$. Let $S \in \mathcal{L} \neq \emptyset$ be an arbitrary losing coalition with $w^{\prime}(S)=q_{1}$. Since

$$
w^{\prime}(N \backslash S)=w^{\prime}(N)-w^{\prime}(S)=1-w^{\prime}(S)=1-q_{1} \leq \frac{1}{2} \leq q_{1}
$$

the complementary coalition $N \backslash S$ would be also losing, which is a contradiction to the assumption that $v$ is strong.

We remark that given a weighted representation of a weighted game $v$, the value of the quota $q \in(0,1]$ is not sufficient to exactly determine whether is proper or non-proper. Similarly $q$ is not sufficient to exactly determine whether is strong or non-strong. As an example we consider the weighted game $[2 ; 1,1,1]$. For each $q \in\left(\frac{1}{3}, \frac{2}{3}\right]$ the representation $\left[q ; \frac{1}{3}, \frac{1}{3}, \frac{1}{3}\right]$ gives the same game.

Lemma 3. A weighted game $v$ is constant-sum if and only if there exists an $\varepsilon>0$ such that for all $q \in\left(\frac{1}{2}-\varepsilon, \frac{1}{2}+\varepsilon\right)$ there exists a normalized weighted representation with quota $q$.

PROOF. If there exists a weighted representation of $v$ with $q=\frac{1}{2}$, then $v$ is strong. If there exists a weighted representation of $v$ with $q=\frac{1}{2}+\frac{\varepsilon}{2}>\frac{1}{2}$, then $v$ is proper.

For the other direction we assume that $v$ is constant-sum. Let $[q ; w]$ we an arbitrary weighted representation of $v$, where $\|w\|_{1}=1$. From Definition 1 we conclude $w \neq 0$, so that we can set $w^{\prime}=\frac{w}{\|w\|_{1}}$, i.e., we normalize the weights to sum 1 . Next we set

$$
q_{1}=\max _{S \in \mathcal{L}} w^{\prime}(S) \quad \text { and } \quad q_{2}=\max _{S \in \mathcal{W}} w^{\prime}(S)
$$

where obviously $0 \leq q_{1}<q_{2} \leq 1$ due to the definition of a weighted game. Each choice of $q^{\prime} \in\left(q_{1}, q_{2}\right]$ corresponds to the same weighted game. From the proofs of Lemma 1 and Lemma 2 we conclude $q_{2}>\frac{1}{2}$ and $q_{1}<\frac{1}{2}$. This obviously admits the choice of a suitable $\varepsilon>0$.

In order to measure the individual's ability to influence the aggregated group decision by changing its own vote, a vast amount of so-called power indices was introduced, see, e.g., [25]. A common core is captured by:

Definition 10. Let $\mathcal{V}_{n} \subseteq \mathcal{B}_{n}$ a class of Boolean games consisting of $n$ voters. A power index (on $\left.\mathcal{V}_{n}\right)$ is a mapping $P: \mathcal{V}_{n} \rightarrow \mathbb{R}^{n}$.

Power indices may have several nice properties:

Definition 11. Let $g: \mathcal{V}_{n} \rightarrow \mathbb{R}^{n}=\left(g_{i}\right)_{i \in N}$ be a power index on a class $\mathcal{V}_{n}$ of Boolean games. We say that

(1) $g$ is symmetric: if for all $v \in \mathcal{V}_{n}$ and any bijection $\tau: N \rightarrow N$ we have $g_{\tau(i)}(\tau v)=g_{i}(v)$, where $\tau v(S)=v(\tau(S))$ for any coalition $S \subseteq N$; 
(2) $g$ is positive: if for all $v \in \mathcal{V}_{n}$ and all $i \in N$ we have $g_{i}(v) \geq 0$ and $g(v) \neq 0$;

(3) $g$ is efficient: iffor all $v \in \mathcal{V}_{n}$ we have $\sum_{i=1}^{n} g_{i}(v)=1$;

(4) g satisfies the null voter property: if for all $v \in \mathcal{V}_{n}$ and all null voters $i$ of $v$ we have $g_{i}(v)=0$.

Examples of power indices for simple games are, e.g., the Shapley-Shubik index [26]

$$
\operatorname{SSI}_{i}(v):=\sum_{S \subseteq N \backslash\{i\}} \frac{|S| !(|N|-1-|S|) !}{|N| !} \cdot(v(S \cup\{i\})-v(S))
$$

and the absolute Banzhaf index [27]

$$
\operatorname{BZI}_{i}(v):=\frac{1}{2^{n-1}} \cdot \sum_{S \subseteq N \backslash\{i\}}(v(S \cup\{i\})-v(S))
$$

for all $i \in N$.

We remark that both indices are symmetric, positive and satisfy the null voter property on $\mathcal{B}_{n}$. The Shapley-Shubik index is efficient on $\mathcal{S}_{n}$, while it is generally not efficient on $\mathcal{B}_{n}$. Whenever a given positive power index $P: \mathcal{V}_{n} \rightarrow \mathbb{R}^{n}$ is not efficient, we can consider its normalization $P_{i}(v) / \sum_{j=1}^{n} P_{j}(v)$.

Boolean games were further generalized:

Definition 12. A coalitional game is a function $v: 2^{N} \rightarrow \mathbb{R}$ with $v(\emptyset)=0$.

Definition 13. Let $\mathcal{V}_{n}$ be a subclass of coalitional games consisting of $n$ voters. A value (on $\left.\mathcal{V}_{n}\right)$ is a mapping $P: \mathcal{V}_{n} \rightarrow \mathbb{R}^{n}$.

Several of the classical power indices have a more general definition as a value. Of course values may also have some of the properties defined in Definition 11. Additionally values can be linear, i.e., we have

$$
P\left(\lambda_{1} \cdot v_{1}+\lambda_{2} \cdot v_{2}\right)=\lambda_{1} \cdot P\left(v_{1}\right)+\lambda_{2} \cdot P\left(v_{2}\right)
$$

for all $\lambda_{1}, \lambda_{2} \in \mathbb{R}$ and all coalitional games $v_{1}, v_{2} \in \mathcal{V}_{n}$.

We complete this section with the definition of the third power index, which is studied and generalized in this paper. To this end let $v$ be a simple game. We call a vector $x \in \mathbb{R}_{\geq 0}^{n}$ with $x(N)=1$ an imputation. The excess of a coalition $S$ for imputation $x$ (in $v$ ) is given by $e(S, x)=v(S)-x(S)$. Let $S_{1}, \ldots, S_{2^{n}}$ be an ordering of all coalitions such that the excess at $x$ is weakly decreasing. The excess vector is the vector $E(x)=\left(e\left(x, S_{k}\right)\right)_{1 \leq k \leq 2^{n}}$. Imputation $x$ is lexicographically less than imputation $y$ if $E_{k}(x)<E_{k}(y)$ for the smallest component $k$ with $E_{k}(x) \neq E_{k}(y)$. With this the nucleolus is then uniquely defined as the lexicographically minimal imputation, see, e.g., [28]. For the weighted game $[3 ; 2,1,1,1]$ the nucleolus is given by $\frac{1}{5} \cdot(2,1,1,1)$, i.e., it coincides with the normalized given weighted representation. The nucleolus has been proposed as a power index, e.g., in $[5,29,30]$. 


\section{3. $(j, k)$ Simple Games}

In this section we briefly introduce the concept of $(j, k)$ (simple) games, mainly based on [8]. So let $j, k \geq 2$ be two arbitrary integers. By $J=\{1, \ldots, j\}$ we denote the alternative options in the input and by $K=\{1, \ldots, k\}$ the alternatives in the output. A numeric evaluation (of the output, i.e., the aggregated group decision) is a function $\alpha: K \rightarrow \mathbb{R}$ with $\alpha(i)>\alpha(i+1)$ for all $1 \leq i<k$. Boolean games, as defined in the previous section, can be seen as $(2,2)$-games, where $J=K=\{1,2\}$ and $\alpha(1)=1, \alpha(2)=0$. In the following we assume the uniform numeric evaluation $\alpha(i)=k-i$ and specify all subsequent definitions without $\alpha$.

Definition 14. A sequence $S=\left(S_{1}, \ldots, S_{j}\right)$ of mutually disjoint sets $S_{i} \subseteq N$ with $\cup_{1 \leq i \leq j} S_{i}=N$ is called ordered j-partition.

For the binary case $j=2$, the set $S_{1}$ is given by the "yes"-voters and $S_{2}$ by the "no"-voters. By $J^{N}$ we denote the set of all ordered $j$-partitions of $N$, i.e., we especially have $\left|J^{N}\right|=j^{n}$. An ordered $j$-partition $S=\left(S_{1}, \ldots, S_{j}\right)$ can also be written as a mapping $\beta: N \rightarrow J$, where $S_{h}=\{i \in N: \beta(i)=h\}$ for all $1 \leq h \leq j$.

Definition 15. For two ordered j-partitions $S=\left(S_{1}, \ldots, S_{j}\right), T=\left(T_{1}, \ldots, T_{j}\right)$ we write $S \stackrel{j}{\subseteq} T$ if

$$
\cup_{1 \leq h \leq i} S_{i} \subseteq \cup_{1 \leq h \leq i} T_{i}
$$

for all $1 \leq i \leq j$.

Definition 16. A Boolean $(\mathbf{j}, \mathbf{k})$ game is given by a function $v: J^{N} \rightarrow K$ with $v((\emptyset, \ldots, \emptyset, N))=k$ and $v((N, \emptyset, \ldots, \emptyset))=1$.

In other words Definition 16 says that if all voters are in favor of the lowest alternative, then the aggregated group decision should be the lowest alternative and similarly for the highest alternative.

Definition 17. A $(\mathbf{j}, \mathbf{k})$ simple game is a Boolean game $v: J^{N} \rightarrow K$ such that $v(S) \leq v(T)$ for all ordered j-partitions $S \stackrel{j}{\subseteq} T$.

Let us consider the following example (taken from [31]) of a $(3,2)$ simple game $v$ given by

$$
\begin{array}{ll}
v\left(S_{1}, S_{2}, S_{3}\right)=2 & \forall S_{1} \subseteq N \backslash\{1\} \\
v\left(S_{1}, S_{2}, S_{3}\right)=2 & \forall\{2,3\} \subseteq S_{3} \subseteq N \\
v\left(S_{1}, S_{2}, S_{3}\right)=1 & \forall\{1\} \subseteq S_{1} \subseteq N,\left|S_{3} \cap\{2,3\}\right| \leq 1
\end{array}
$$

where $S_{1}, S_{2}, S_{3}$ form an ordered 3-partition of $N=\{1,2,3\}$. In other words the aggregated group decision is 1 if and only if voter 1 is in favor of alternative 1 and not both of the remaining voters are in favor of alternative 3 .

We remark that there are also notions for complete or weighted $(j, k)$ games, but according to [16] no completely satisfactory definition of a weighted $(j, k)$ game has been found so far, while several suggestions have been proposed in the literature.

In order to state the Shapley-Shubik and the Banzhaf index for $(j, k)$ simple games, see [15,16], we need a few further definitions. 
Definition 18. A queue of $N=\{1, \ldots, n\}$ is a bijection from $N$ to $N$. The set of all queues is denoted by $\mathcal{Q}_{n}$, i.e., $\left|\mathcal{Q}_{n}\right|=n$ !.

Definition 19. Let $v: J^{N} \rightarrow K$ be a $(j, k)$ simple game, $q \in \mathcal{Q}_{n}$ a queue, and $S=\left(S_{1}, \ldots, S_{j}\right)$ an ordered $j$-partition. For each $1 \leq i \leq k-1$ the i-pivot is uniquely defined either as

(1) the voter, whose vote in $S$ clinches the aggregated group decision under, at least the output level $i$, independently of the subsequent voters of $i$ in $q$, or

(2) the voter, whose vote in $S$ clinches the aggregated group decision under, at most the output level $i+1$, independently of the subsequent voters of $i$ in $q$.

For the example of the $(3,2)$ game $v$ above, we consider the queue $q=(2,1,3)$ and the ordered 3 -partition $S=(\{1\},\{2\},\{3\})$. Since $v(\{1,3\},\{2\}, \emptyset)=1$ and $v(\emptyset,\{2\},\{1,3\})=2$, voter 2 is not a 1-pivot for $q$ and $S$ in $v$. Since $v(\{1\},\{2\},\{3\})=v(\{1\},\{2,3\}, \emptyset)=v(\{1,3\},\{2\}, \emptyset)=1$, voter 1 is not a 1-pivot for $q$ and $S$ in $v$ and voter 3 is a 1-pivot for $q$ and $S$ in $v$.

Definition 20. The Shapley-Shubik index of a $(j, k)$ simple game is given by

$$
\operatorname{SSI}_{i}(v)=\frac{1}{n ! \cdot j^{n}} \cdot \sum_{h=1}^{k-1} \mid\left\{(q, S) \in \mathcal{Q}_{n} \times J^{N}: i \text { is a h-pivot for } q \text { and } S \text { in } v\right\} \mid
$$

for all $i \in N$.

For the stated example of the $(3,2)$ simple game we obtain the following pivot-counts per permutation:

$$
\begin{array}{rlr}
(1,2,3) \rightarrow(18,6,3) & (2,1,3) \rightarrow(24,0,3) & (3,1,2) \rightarrow(24,3,0) \\
(1,3,2) \rightarrow(18,3,6) & (2,3,1) \rightarrow(24,0,3) & (3,2,1) \rightarrow(24,3,0)
\end{array}
$$

so that $\operatorname{SSI}(v)=\left(\frac{22}{27}, \frac{5}{54}, \frac{5}{54}\right)$.

Definition 21. Given a $(j, k)$ simple game $v$ and an ordered $j$-partition $S \in J^{N}$, we denote by $S_{i \downarrow}$ the unique ordered $j$ partition which satisfies

(1) $S_{h} \backslash\{i\}=T_{h} \backslash\{i\}$ for all $1 \leq h \leq j$ and

(2) $i \in T_{\max (h+1, j)}$ for the index $h$ with $i \in S_{h}$,

where we use the abbreviation $T=S_{i \downarrow}$. The pair $\left(S, S_{i \downarrow}\right) \in J^{N} \times J^{N}$ is called an (m, $\left.\mathbf{l}\right)$-swing for voter $\mathrm{i}$ if $1 \leq l<m \leq k, v(S)=l$, and $v(T)=m$. The number of all $(m, l)$-swings for voter $i$ in $v$ is denoted by $\eta_{i}(v)$.

Definition 22. The absolute Banzhaf index of a $(j, k)$ simple game is given by

$$
\mathrm{BZI}_{i}(v)=\frac{1}{j^{n-1} \cdot(k-1)} \cdot \eta_{i}(v)
$$

for all $i \in N$. 
We remark that the normalization factor $\frac{1}{j^{n-1} \cdot(k-1)}$, which is not contained in the definition stated in [15], is rather debatable, but this way our definitions coincide with the usual definitions for $(2,2)$ simple games. In most applications the absolute Banzhaf index is normalized to be efficient anyway.

For the stated example of the $(3,2)$ simple game we obtain $\eta_{1}(v)=8, \eta_{2}(v)=\eta_{3}(v)=1$, so that $\mathrm{BZI}=\frac{1}{9} \cdot(8,1,1)$ and that the normalized Banzhaf index is given by $\left(\frac{4}{5}, \frac{1}{10}, \frac{1}{10}\right)$. We remark that for this specific example the $\|\cdot\|_{1}$-norm of the difference of the Shapley-Shubik index and the normalized Banzhaf index is given by $\frac{4}{135} \approx 3 \%$.

To the best of our knowledge the nucleolus has not been defined for $(j, k)$ simple games so far. In Section 6 we will extend the definition of the nucleolus of simple games to continuous decision rules. The underlying, rather natural idea, can be used to define the nucleolus also for $(j, k)$ simple games.

For the special case of $(3,2)$ simple games some more power indices were defined in [32].

\section{Continuous Decision Rules}

In order to rewrite the definitions and results of Section 2 for the continuous interval $[0,1]$ instead of the binary set $\{0,1\}$ of alternatives, we identify $2^{N}$ with $\{0,1\}^{n}$, i.e., subsets are mapped to incidence vectors

$$
S \subseteq N \mapsto\left(x_{1}, \ldots, x_{n}\right) \in\{0,1\}^{n}
$$

where $x_{i}=1$ if $i \in S$ and $x_{i}=0$ otherwise. An example is given by the incidence vector $(1,0,1,0)$ for the coalition $\{1,3\} \subseteq\{1,2,3,4\}$.

Definition 23. A continuous Boolean game is a function $v:[0,1]^{n} \rightarrow[0,1]$ with $v((0, \ldots, 0))=0$ and $v((1, \ldots, 1))=1$. The set of all continuous Boolean games on $n$ players is denoted by $\mathbb{B}_{n}$.

We remark that requiring $v((x, \ldots, x))=x$ for all $x \in[0,1]$ would be a rather strong condition, which is violated by several of the examples considered later on. ${ }^{2}$

Each Boolean game $v$ can be embedded in a continuous Boolean game $v^{\prime}$ extending the function defined on the $2^{n}$ points $\{0,1\}^{n}$ in an arbitrary way. If $v$ is linear, i.e., if $v(S \cup T)=v(S)+v(T)$ for all $S, T \subseteq N$ with $S \cap T$, then we can use convex combinations of the $2^{n}$ points $\{0,1\}^{n}$ to interpolate the intermediate points in $[0,1]^{n}$. Another way is to use a partition $S, T$ of $[0,1]$ in the following way: For each $x^{\prime} \in[0,1]^{n}$ we define $x \in\{0,1\}^{n}$ by $x_{i}=0$ if $x_{i}^{\prime} \in S$ and $x_{i}=1$ if $x_{i}^{\prime} \in T$. With this we can set $v^{\prime}\left(x^{\prime}\right)=v(x) \in\{0,1\}$. We remark that using this threshold-type embedding the definitions stated in this section are transfered back to those from Section 2.

Instead of winning and losing coalitions we can look more generally at $z$-coalitions $\left\{i \in N \mid x_{i}=z\right\}$, $\underline{z}$-coalitions $\left\{i \in N \mid x_{i} \leq z\right\}$, and $\bar{z}$-coalitions $\left\{i \in N \mid x_{i} \geq z\right\}$ for each $z \in[0,1]$. So winning coalitions are 1 -coalitions and losing coalitions are 0 -coalitions if all $x_{i}$ are binary.

Definition 24. For two vectors $x=\left(x_{1}, \ldots, x_{n}\right) \in \mathbb{R}^{n}$ and $y=\left(y_{1}, \ldots, y_{n}\right) \in \mathbb{R}^{n}$ we write $x \leq y$ if $x_{i} \leq y_{i}$ for all $1 \leq i \leq n$.

2 In some applications one may consider the mentioned condition as a natural requirement. As an example consider the players' input $x$ as their most preferred alternative. If all players unanimously prefer $x$ to all other alternatives, then $x$ clearly should be implemented. 
Definition 25. A continuous simple game is a continuous Boolean game $v:[0,1]^{n} \rightarrow[0,1]$ such that $v(S) \leq v(T)$ for all real-valued vectors $0 \leq S \leq T \leq 1$, where $\mathbf{0}$ denotes the all-0-and 1 the all-1-vector. The set of all continuous simple games on $n$ players is denoted by $\mathbb{S}_{n}$.

Definition 26. Given a continuous Boolean $v:[0,1]^{n} \rightarrow[0,1]$, each voter $i \in N$ such that

$$
v\left(\left(x_{1}, \ldots, x_{n}\right)\right)=v\left(\left(x_{1}, x_{i-1}, x_{i}^{\prime}, x_{i+1}, \ldots, x_{n}\right)\right)
$$

for all $x_{1}, \ldots, x_{n}, x_{i}^{\prime} \in[0,1]$ is called a null voter.

We remark that Definition 26 is equivalent to Definition 4 if all $x_{i}$ are binary.

Definition 27. A continuous simple game $v:[0,1]^{n} \rightarrow[0,1]$ is called proper if $v(x)+v(\mathbf{1}-x) \leq 1$ for all real-valued vectors $x \in[0,1]^{n}$. It is called strong if $v(x)+v(1-x) \geq 1$. A continuous simple game that is both proper and strong is called constant-sum (or self-dual, or decisive).

In analogy to Definition 6 we generalize Isbell's desirability relation as follows:

Definition 28. Given a continuous simple game $v:[0,1]^{n} \rightarrow[0,1]$ we say that voter $i \in N$ is more desirable as voter $j \in N$, denoted by $i \succeq j$, if

(1) $v(\tau(x)) \geq v(x)$ for all $x \in[0,1]^{n}$ with $x_{i} \leq x_{j}$, where $\tau$ is equal to the transposition $(i, j)$;

(2) $v(\tau(x)) \leq v(x)$ for all $x \in[0,1]^{n}$ with $x_{i} \geq x_{j}$, where $\tau$ is equal to the transposition $(i, j)$.

We write $i \simeq j$ if $i \succeq j, j \succeq i$ and use $i \succ j$ as abbreviation for $i \succeq j, i \not z j$.

We can easily check that Definition 28 is equivalent to Definition 6 if all $x_{i}$ are binary.

Definition 29. A continuous simple game $v:[0,1]^{n} \rightarrow[0,1]$ is called complete iffor each pair of voters $i, j \in N$, we have $i \succeq j$ or $j \succeq i$. The set of all continuous complete (simple) games on $n$ voters is denoted by $\mathbb{C}_{n}$.

As mentioned in the previous section, no completely satisfactory definition of a weighted $(j, k)$ game has been found so far, so that we propose several versions of weightedness in the case of continuous simple games.

Definition 30. A continuous simple game $v:[0,1]^{n} \rightarrow[0,1]$ is linearly weighted, if there exist (normalized) weights $w_{1}, \ldots, w_{n} \geq 0$ with $\sum_{i=1}^{n} w_{i}=1$, such that $v\left(\left(x_{1}, \ldots, x_{n}\right)\right)=\sum_{i=1}^{n} w_{i} x_{i}$. The set of all continuous linearly weighted (simple) games on $n$ voters is denoted by $\mathbb{L}_{n}$.

Definition 31. A continuous simple game $v:[0,1]^{n} \rightarrow[0,1]$ is called a threshold game if there exists a quota $q \in(0,1]$ and (normalized) weights $w_{1}, \ldots, w_{n} \geq 0$ with $\sum_{i=1}^{n} w_{i}=1$ such that $v\left(\left(x_{1}, \ldots, x_{n}\right)\right)=1$ if $\sum_{i=1}^{n} w_{i} x_{i} \geq q$ and $v\left(\left(x_{1}, \ldots, x_{n}\right)\right)=0$ otherwise. The set of all continuous threshold games on $n$ voters is denoted by $\mathbb{T}_{n}$.

Definition 32. A continuous simple game $v:[0,1]^{n} \rightarrow[0,1]$ is weighted if there exist (normalized) weights $w_{1}, \ldots, w_{n} \geq 0$ with $\sum_{i=1}^{n} w_{i}=1$ and a monotonously increasing quota function $q:[0,1] \rightarrow[0,1]$ such that $v\left(\left(x_{1}, \ldots, x_{n}\right)\right)=q\left(\sum_{i=1}^{n} w_{i} x_{i}\right)$. The set of all continuous weighted ( simple) games on $n$ voters is denoted by $\mathbb{W}_{n}$. 
The quota function $q$ of a weighted continuous simple game satisfies $q(0)=0$ and $q(1)=1$. We remark that all linearly weighted, all threshold, and all weighted continuous simple games are complete.

Lemma 4. The weighted representation of a continuous linearly weighted game is unique.

PROOF. Assume that a given continuous linearly weighted game $v$ has two weighted representations $w$ and $\hat{w}$ with $w, \hat{w} \in[0,1]^{n}$ and $\|w\|_{1}=\|\hat{w}\|_{1}=1$. Thus we have $v(x)=w^{T} x=\hat{w}^{T} x$ for all $x \in[0,1]^{n}$. Inserting $x=(0, \ldots, 0,1,0, \ldots, 0)$, where the 1 is at position $1 \leq i \leq n$, yields $w_{i}=\hat{w}_{i}$, so that $w=\hat{w}$.

Lemma 5. Let $v$ be a continuous threshold game such that there exists a vector $x \neq 1$ with $v(x)=1$ and $x_{j}<1$ for a non-null voter $j$. The weighted representation of $v$, consisting of a quota $q \in(0,1]$ and weights $w \in[0,1]^{n}$ with $\|w\|_{1}=1$, is unique.

PROOF. Let $\left(q^{(1)}, w^{(1)}\right)$ and $\left(q^{(2)}, w^{(2)}\right)$ be two representations of $v$, i.e.,

$$
\sum_{i=1}^{n} w_{i}^{(1)} \tilde{x}_{i} \geq q^{(1)} \Longleftrightarrow \sum_{i=1}^{n} w_{i}^{(2)} \tilde{x}_{i} \geq q^{(2)}
$$

for all $\tilde{x} \in[0,1]^{n}$. Since $q^{(1)} \in[0,1]$ there exists a $\hat{x} \in[0,1]^{n}$ with $\hat{x}^{T} w^{(1)}=q^{(1)}$. Each voter $1 \leq i \leq n$ with $w_{i}^{(1)}=0$ or $w_{i}^{(2)}=0$ is a null voter.

Assume $q^{(1)}=1$ : For $x \neq \mathbf{1}$ there is a non-null voter $1 \leq j \leq n$ with $x_{j}<1$. Thus $w_{j}^{(1)}=0$ since otherwise $x^{T} w^{(1)}<1$, which is contradictory to $j$ being a non-null voter. Thus we have $q^{(1)} \neq 1$.

Next we show that each null voter has zero weight. Let $e_{j}$ denote the $j$-th unit vector and let $i$ be a null voter. If $\hat{x}_{i}>0$ then $w^{(1)}\left(\hat{x}-\varepsilon \cdot e_{i}\right)<q^{(1)}$ for all $\varepsilon>0$, so that $v\left(\hat{x}-\varepsilon \cdot e_{i}\right)=0$ (for suitably small $\varepsilon$ ), which is contradictory to $i$ being a null voter. If otherwise $\hat{x}_{i}=0$ for all null voters $i$, then there exists a non-null voter $j$ with $\hat{x}_{j}>0$ and $w_{j}^{(1)}>0$. With this we have $w^{(i)}\left(\hat{x}-\varepsilon \cdot e_{j}\right)<q^{(1)}$ for $\varepsilon>0$ and $v\left(\hat{x}-\varepsilon \cdot e_{j}\right)=0$ (for suitably small $\varepsilon$ ). Since $w^{(1)}\left(\hat{x}-\varepsilon \cdot e_{j}+\varepsilon \cdot \frac{w_{j}^{(1)}}{w_{i}^{(1)}} \cdot e_{i}\right)=q^{(1)}$, where $i$ is an arbitrary null voter, we have $v\left(\hat{x}-\varepsilon \cdot e_{j}+\varepsilon \cdot \frac{w_{j}^{(1)}}{w_{i}^{(1)}} \cdot e_{i}\right)=1$, which contradicts the fact that $i$ is a null voter. Thus each null voter has zero weight.

Due to symmetry we can state $q^{(1)}, q^{(2)} \in(0,1), w_{j}^{(1)}, w_{j}^{(2)} \in(0,1]$ for all non-null voters $j$, and $w_{i}^{(1)}=w_{i}^{(2)}=0$ for all null voters $i$. If there exists exactly one non-null voter $j$ in $v$, then we have $w_{j}^{(1)}=1=w_{j}^{(2)}$ due to $\left\|w^{(1)}\right\|_{1}=\left\|w^{(2)}\right\|_{1}=1$, so that $w^{(1)}=w^{(2)}$. Thus we assume that the number of non-null voters is at least two and $w_{j}^{(1)}, w_{j}^{(2)} \in(0,1)$ for all non-null voters $j$ in the following.

We have $v(\hat{x})=1$ and $v\left(\hat{x}-\varepsilon \cdot e_{j}\right)=0$, where $1 \leq j \leq n$ is a non-null voter and $\varepsilon>0$ is arbitrary (but suitably small). Thus we have $q^{(2)}=q^{(1)}$. For two arbitrary non-null voters indices $1 \leq j_{1}, j_{2} \leq n$ and suitably small $\varepsilon>0$ we have

$$
q^{(1)}=w^{(1)}\left(\hat{x}-w_{j_{2}}^{(1)} \cdot \varepsilon \cdot e_{j_{1}}+w_{j_{1}}^{(1)} \cdot \varepsilon \cdot e_{j_{2}}\right)=w^{(2)}\left(\hat{x}-w_{j_{2}}^{(1)} \cdot \varepsilon \cdot e_{j_{1}}+w_{j_{1}}^{(1)} \cdot \varepsilon \cdot e_{j_{2}}\right)=q^{(2)}
$$

Thus

$$
\frac{w_{j_{1}}^{(1)}}{w_{j_{2}}^{(1)}}=\frac{w_{j_{1}}^{(2)}}{w_{j_{2}}^{(2)}}
$$

and we conclude $w^{(1)}=w^{(2)}$ from $\left\|w^{(1)}\right\|_{1}=\left\|w^{(2)}\right\|_{1}=1$ and $w_{i}^{(1)}=w_{i}^{(2)}=$ for the null voters $i$. 
For the case of quota $q=1$ we remark, that any (normalized) weight vector $w \in[0,1]^{n}$ leads to the same continuous threshold game.

Given a finite number $k$ of continuous threshold games $v_{1}, \ldots, v_{k}$ we call the game arising by

$$
v(x)=\min _{1 \leq i \leq k}\left\{v_{i}(x)\right\}
$$

the intersection of the continuous threshold games $v_{i} . v$ is indeed a continuous simple game, but not every continuous simple game can be written as a finite intersection of continuous threshold games. An example is given by the continuous simple game $v$ with $v(x)=1$ if $\frac{1}{n} \cdot \sum_{i=1}^{n} x_{i}^{2} \geq \frac{1}{2}$ and $v(x)=0$ otherwise. Here infinitely many continuous threshold games are needed in the intersection.

Lemma 6. The quota function $q$ of a continuous weighted game $v:[0,1]^{n} \rightarrow[0,1]$ is unique. If $q$ is monotone and continuous, then also the weights $w_{i}$ are unique.

PROOF. Since $v(x, \ldots, x)=q(x)$ for all $x \in[0,1]$ the quota function is uniquely defined.

Since $q(0)=0, q(1)=1, q$ is monotone and continuous, there exists a value $x \in[0,1]$ such that $y=q^{-} 1(x) \in(0,1)$ is uniquely defined. If $v(y, \ldots, y, y+\varepsilon, y, \ldots, y)=v(y, \ldots, y)$ for $\varepsilon=\min (y, 1-y) / 2$ and a modified position $1 \leq i \leq n$, then $w_{i}=0$. For all other positions we have $w_{j}>0$. Let $i$ and $j$ be two positions with $w_{i}, w_{j}>0$. For suitably small $\varepsilon>0$ there exists a unique $\delta>0$ such that $v(z)=\operatorname{game}(y, \ldots, y)$, where $z_{i}=y+\varepsilon, z_{j}=y-\delta$, and $z_{h}=y$ for $h \neq i, j$. From this we conclude $\varepsilon w_{i}=\delta w_{j}$, so that we can uniquely determine all $w_{h}$ due to $\|w\|_{1}=1$.

So, depending on the chosen definition of weightedness, the corresponding weighted representations are either unique or not. For the different versions of weightedness the connection to the properties proper, strong, and constant-sum is as follows:

Lemma 7. All continuous linearly weighted games are proper, strong, and constant-sum.

PROOF. For a given continuous weighted game $v$ let $w \in[0,1]^{n}$ with $\|w\|_{1}=1$ be suitable (normalized) weights. With this we have

$$
v(x)+v(\mathbf{1}-x)=w^{T} x+w^{T}(\mathbf{1}-x)=\|w\|_{1}=1
$$

for all $x \in[0,1]^{n}$.

Lemma 8. A continuous threshold game $v$ with (normalized) weights $w$ and quota $q \in(0,1]$ is proper if and only if $q>\frac{1}{2}$.

PROOF. Since

$$
v\left(\frac{1}{2} \cdot \mathbf{1}\right)=\frac{1}{2} w^{T} \mathbf{1}=1-\frac{1}{2} w^{T} \mathbf{1}=v\left(\mathbf{1}-\frac{1}{2} \cdot \mathbf{1}\right)
$$

the game $v$ is non-proper for $q \leq \frac{1}{2}$. Now assume $q>\frac{1}{2}$. We have $v(x) \in\{0,1\}$ for all $x \in[0,1]^{n}$. Assume that both $v(x)=1$ and $v(\mathbf{1}-x)=1$. Then we would have $w^{T} x \geq q$ and $w^{T}(\mathbf{1}-x) \geq q$ so that

$$
1=\|w\|_{1} \geq 2 q
$$

which is a contradiction to $q>\frac{1}{2}$. 
Lemma 9. A continuous threshold game $v$ with (normalized) weights $w$ and quota $q \in(0,1]$ is strong if and only if $q \leq \frac{1}{2}$.

Proof. Since

$$
v\left(\frac{1}{2} \cdot \mathbf{1}\right)=\frac{1}{2} w^{T} \mathbf{1}=1-\frac{1}{2} w^{T} \mathbf{1}=v\left(\mathbf{1}-\frac{1}{2} \cdot \mathbf{1}\right)
$$

the game $v$ is non-strong for $q>\frac{1}{2}$. Now assume $q \leq \frac{1}{2}$. We have $v(x) \in\{0,1\}$ for all $x \in[0,1]^{n}$. Assume that both $v(x)=0$ and $v(\mathbf{1}-x)=0$. Then we would have $w^{T} x<q$ and $w^{T}(\mathbf{1}-x)<q$ so that

$$
1=\|w\|_{1}<2 q
$$

which is a contradiction to $q \leq \frac{1}{2}$.

Corollary 1. No continuous threshold game can be constant-sum.

Lemma 10. A continuous weighted game $v$ with (normalized) weights $w$ and quota function $q:[0,1] \rightarrow$ $[0,1]$ is proper if and only if $q(y)+q(1-y) \leq 1$ for all $y \in[0,1]$.

PROOF. For arbitrary $x \in[0,1]^{n}$ we have

$$
v(x)+v(\mathbf{1}-x)=q\left(w^{T} x\right)+q\left(w^{T}(\mathbf{1}-x)\right)=q(y)+q(1-y) \leq 1
$$

where $y=w^{T} x \in[0,1]$.

Lemma 11. A continuous weighted game $v$ with (normalized) weights $w$ and quota function $q:[0,1] \rightarrow$ $[0,1]$ is strong if and only if $q(y)+q(1-y) \geq 1$ for all $y \in[0,1]$.

PROOF. For arbitrary $x \in[0,1]^{n}$ we have

$$
v(x)+v(\mathbf{1}-x)=q\left(w^{T} x\right)+q\left(w^{T}(\mathbf{1}-x)\right)=q(y)+q(1-y) \geq 1
$$

where $y=w^{T} x \in[0,1]$.

Corollary 2. A continuous weighted game $v$ with (normalized) weights $w$ and quota function $q:[0,1] \rightarrow$ $[0,1]$ is constant-sum if and only if $q(y)+q(1-y)=1$ for all $y \in[0,1]$.

The notion of a power index can be transfered as follows:

Definition 33. Let $\mathbb{V}_{n} \subseteq \mathbb{B}_{n}$ a class of continuous Boolean games consisting of $n$ voters. A power index $\left(\right.$ on $\mathbb{V}_{n}$ ) is a mapping $P: \mathbb{V}_{n} \rightarrow \mathbb{R}^{n}$.

The four properties of power indices for subclasses of Boolean games, see Definition 11, can be restated one to one for power indices for subclasses of continuous Boolean games.

Before we give definitions for the Shapley-Shubik index, the absolute Banzhaf index and the nucleolus for continuous simple games in Section 6, we discuss some special classes of continuous games in the next section. 


\section{Examples of Continuous Games}

The definitions of linearly weighted, threshold, and weighted continuous simple games in the previous section allow a compact representation of those games given a weight vector $w$ and eventually a quota or quota function $q$.

Numerous theoretical models for the behavior of politicians are based on the so-called median voter model, see, e.g., [33-36]. The median voter theorem states that in a voting system with a single majority decision rule, the most probable elected alternative is the one which is most preferred by the median voter. The key assumptions of a 1-dimensional policy space with single peaked preferences are met in our context. So similarly to Hotelling's law, according to the median voter model, politicians try to adjust their opinions near the preferences of the expected median voter. In practice there are several limitations for the median voter theorem so that the explanatory power of the median voter model is actually rather low, see, e.g., [37].

In our context the situation is a bit easier. Given the single peaked preferences $x_{i} \in[0,1]$ of the voters, the aggregated group decision can be any number in $[0,1]$, i.e., we neither have to choose within a finite number of alternatives nor do we indirectly influence future decisions by electing a representative. So it makes quite some sense to utilize the median aggregation rule given by

$$
v\left(x_{1}, \ldots, x_{n}\right)=\left\{\begin{array}{rll}
x_{\pi\left(\frac{n+1}{2}\right)} & : n \equiv 1 & (\bmod 2) \\
\frac{1}{2} \cdot x_{\pi\left(\frac{n}{2}\right)}+\frac{1}{2} \cdot x_{\pi\left(\frac{n+2}{2}\right)} & : n \equiv 0 & (\bmod 2)
\end{array}\right.
$$

where $\pi$ is a permutation such that $x_{\pi(1)} \leq \cdots \leq x_{\pi(n)}$. This decision rule can be slightly generalized by introducing non-negative weights $w_{i}$ for all voters $1 \leq i \leq n$ such that $\|w\|_{1}=\sum_{i=1}^{n} w_{i}>0$. With a permutation $\pi$ as before, let $\underline{i}$ be the smallest index such that $\sum_{j=1}^{i} w_{\pi(j)} \geq\|w\|_{1} / 2$. Similarly, let $\bar{i}$ be the largest index such that $\sum_{j=\bar{i}}^{n} w_{\pi(j)} \geq\|w\|_{1} / 2$. If $\underline{i}=\bar{i}$ we set $v\left(x_{1}, \ldots, x_{n}\right)=x_{\pi(\underline{i})}$ and $v\left(x_{1}, \ldots, x_{n}\right)=\left(x_{\pi(\underline{i})}+x_{\pi(\bar{i})}\right) / 2$ otherwise. We call this procedure the weighted median aggregation rule.

And indeed, continuous games (without our more general notion) equipped with the weighted median aggregation rule are e.g., studied in [1,19-21]. We will see that some formulas for power indices, defined in the subsequent sections, can be significantly simplified for the (weighted) median aggregation rule.

Another source of group aggregation rules is the field of opinion dynamics. Assume that each voter starts with an initial opinion $x_{i} \in[0,1]$ followed by a dynamic process of exchanging opinions between the individuals. Such an opinion dynamics influences the initial opinions in a certain way, so that the opinion $x_{i}^{\prime}$, after some rounds of interaction, may significantly differ from the initial ones. Group aggregation for the final opinions $x_{i}^{\prime}$ may be performed using weighted voting or the median aggregation rule. Several models for opinion dynamics, i.e., specifications how the $x_{i}$ are modified to the $x_{i}^{\prime}$ have been proposed in the literature. Here we only mention the Lehrer-Wagner model, see, e.g., [38], the bounded confidence model, see, e.g., [39], model based on opinion leaders, see, e.g., [40,41], and the more recent models proposed by Grabisch and Rusinowska [18,42-45] (being based on the ground of [46]). An overview of social and economic networks is given in [47]. 


\section{Generalizing Three Power Indices}

In this section we propose generalizations of the Shapley-Shubik index, the Banzhaf index and the nucleolus for continuous simple games, which are, in a certain sense, in line with the definitions for simple games or $(j, k)$-simple games. We illustrate our definitions by computing the respective indices for the functions $\hat{v}\left(x_{1}, x_{2}, x_{3}\right)=\frac{1 x_{1}^{2}+2 x_{2}^{2}+3 x_{3}^{2}}{6}$ and $\tilde{v}\left(x_{1}, x_{2}, x_{3}\right)=x_{1} x_{2}^{2} x_{3}^{3}$.

\subsection{Shapley-Shubik Index}

One interpretation for the definition of the Shapley-Shubik index for simple games is the following:

(1) According to the veil of ignorance, the set of vote vectors has no structure, i.e., votes are independent and each of the $2^{n}\{0,1\}$-vectors occurs with equal probability.

(2) Assume that the voters are arranged in a sequence and called one by one. After the $i$ th voter in the current sequence has expressed his vote, an output alternative may be excluded independently from the votes of the subsequent voters. Here, all sequences are equally probable and the exclusion of an output alternative is counted just once, i.e., it is counted for the first player who excludes it.

Going along the same lines for $(j, k)$ simple games, we have $j^{n}$ possible input vectors in (1) and $n$ ! possible sequences in (2). The notion of an $i$-pivot in Definition 19 exactly determines the voter who excludes output alternative $i$ or $i+1$, where we have to consider the direction of the exclusion to avoid double counting.

Lets look at the highest and the lowest possible outcome of a $(j, k)$ simple game $v$ given the first $i$ votes $x_{1}, \ldots, x_{i}$. Due to monotonicity the highest possible outcome occurs if the remaining $n-i$ voters vote for the highest possible (input) alternative. Similarly, the lowest possible outcome occurs if the remaining $n-i$ voters vote for the lowest possible (input) alternative. For continuous simple games the extremal input alternatives are given by 0 and 1 so that we define:

\section{Definition 34.}

- $\bar{\tau}:[0,1]^{n} \times\{1, \ldots, n\} \rightarrow[0,1]^{n},(x, i) \mapsto\left(y_{1}, \ldots, y_{n}\right)$, where $y_{j}=x_{j}$ for all $1 \leq j \leq i$ and $y_{j}=1$ otherwise;

- $\underline{\tau}:[0,1]^{n} \times\{1, \ldots, n\} \rightarrow[0,1]^{n},(x, i) \mapsto\left(y_{1}, \ldots, y_{n}\right)$, where $y_{j}=x_{j}$ for all $1 \leq j \leq i$ and $y_{j}=0$ otherwise.

With this we can count the number of excluded output alternatives and sum over all possible sequences and vote distributions. Since the output interval $[0,1]$ is continuous, counting here means to measure the length of the newly excluded interval. There are $n !<\infty$ possible sequences of the $n$ voters, so that summing here really means summing up. Since the space $[0,1]^{n}$ of possible vote distributions is continuous we have to utilize integrals: 
Definition 35. Let $v:[0,1]^{n} \rightarrow[0,1]$ be a continuous simple game. The Shapley-Shubik index $\operatorname{SSI}_{i}(v)$ of voter $i$ in $v$ is given by

$$
\begin{aligned}
& \frac{1}{n !} \cdot \sum_{\pi \in \mathcal{S}_{n}} \int_{0}^{1} \ldots \int_{0}^{1}\left(v\left(\bar{\tau}\left(x, \pi^{-1}(i)-1\right)\right)-v\left(\bar{\tau}\left(x, \pi^{-1}(i)\right)\right)\right) \\
& +\left(v\left(\underline{\tau}\left(x, \pi^{-1}(i)\right)\right)-v\left(\underline{\tau}\left(x, \pi^{-1}(i)-1\right)\right)\right) \mathrm{d} x_{1} \ldots \mathrm{d} x_{n}
\end{aligned}
$$

where $\mathcal{S}_{n}$ denotes the symmetric group on $n$ elements, i.e., the set of permutations or bijections from $\{1, \ldots, n\}$ to $\{1, \ldots, n\}$.

For our two examples we obtain

$$
\operatorname{SSI}(\hat{v})=\left(\frac{1}{6}, \frac{2}{6}, \frac{3}{6}\right)=(0.1 \overline{6}, 0 . \overline{3}, 0.5)
$$

and

$$
\operatorname{SSI}(\tilde{v})=\left(\frac{35}{144}, \frac{50}{144}, \frac{59}{144}\right)=(0.2430 \overline{5}, 0.347 \overline{2}, 0.4097 \overline{2})
$$

The detailed computations are stated in Appendix A.

While the story of interpreting the Shapley-Shubik index, stated at the beginning of this section, may be considered to be nice, more serious characterizations involve a so-called axiomatization, see, e.g., [48]:

Lemma 12. Let $P: \mathcal{S}_{n} \rightarrow \mathbb{R}^{n}$ be a power index. If $P$ satisfies symmetry, efficiency, the null voter property, and the transfer axiom, then $P$ coincides with the Shapley-Shubik index.

In order to define the transfer axiom for simple games we need:

Definition 36. For two Boolean games $v_{1}, v_{2} \in \mathcal{S}_{n}$ we define $v_{1} \vee v_{2}$ by $\left(v_{1} \vee v_{2}\right)(S)=$ $\max \left(v_{1}(S), v_{2}(S)\right)$ for all $S \subseteq N$. Similarly, we define $v_{1} \wedge v_{2}$ by $\left(v_{1} \wedge v_{2}\right)(S)=\min \left(v_{1}(S), v_{2}(S)\right)$.

Definition 37. A power index $P: \mathcal{V}_{n} \rightarrow \mathbb{R}^{n}$ satisfies the transfer axiom, if

$$
P\left(v_{1}\right)+P\left(v_{2}\right)=P\left(v_{1} \wedge v_{2}\right)+P\left(v_{1} \vee v_{2}\right)
$$

for all $v_{1}, v_{2} \in \mathcal{V}_{n}$ such that also $\left(v_{1} \wedge v_{2}\right),\left(v_{1} \vee v_{2}\right) \in \mathcal{V}_{n}$, where $\mathcal{V}_{n}$ is a subclass of (binary) Boolean games.

Definition 37 can be restated directly for continuous Boolean games using:

Definition 38. For two continuous Boolean games $v_{1}, v_{2}$ we define $v_{1} \vee v_{2}$ by $\left(v_{1} \vee v_{2}\right)(x)=$ $\max \left(v_{1}(x), v_{2}(x)\right)$ for all $x \in[0,1]^{n}$. Similarly, we define $v_{1} \wedge v_{2}$ by $\left(v_{1} \wedge v_{2}\right)(x)=\min \left(v_{1}(x), v_{2}(x)\right)$.

If $v_{1}, v_{2} \in \mathbb{S}_{n}$, then also $\left(v_{1} \wedge v_{2}\right),\left(v_{1} \vee v_{2}\right) \in \mathbb{S}_{n}$. Directly from the definitions we conclude:

Lemma 13. The Shapley-Shubik index SSI is symmetric, positive, and satisfies both the null voter property and the transfer axiom on $\mathbb{S}_{n}$. 
Conjecture 1. The Shapley-Shubik index for continuous simple games is efficient, i.e., $\sum_{i=1}^{n} \operatorname{SSI}_{i}(v)=1$ for all $v \in \mathbb{S}_{n}$.

Conjecture 2. Let $P: \mathbb{S}_{n} \rightarrow \mathbb{R}^{n}$ be a power index. If $P$ satisfies symmetry, efficiency, the null voter property, and the transfer axiom, then $P$ coincides with the Shapley-Shubik index according to Definition 35.

As remarked before, the formula for the Shapley-Shubik index can be simplified for the weighted median aggregation rule. To this end let $w \in \mathbb{R}_{\geq 0}^{n}$ be a weight vector with $\|w\|_{1}>0$. To avoid technical difficulties we assume $\sum_{i \in S} w_{i} \neq\|w\|_{1} / 2$ for all $S \subseteq N$, i.e., that there is always a unique weighted median voter. Without proof we state:

Lemma 14. The Shapley-Shubik index of the weighted median aggregation rule, according to Definition 35 is given by the Shapley-Shubik index of the weighted game $\left[\|w\|_{1} / 2 ; w_{1}, \ldots, w_{n}\right]$.

We give an example in Appendix B.

\subsection{Banzhaf Index}

One interpretation for the definition of the Banzhaf index for simple games and $(j, k)$ simple games is the following:

(1) According to the veil of ignorance, the set of vote vectors has no structure, i.e., votes are independent and each of the $j^{n} J$-vectors occurs with equal probability.

(2) Relevant for the measurement of influence is only the number of $(m, l)$-swings (or swings for simple games) for voter $i$ arising if voter $i$ shifts his chosen alternative by one.

For continuous simple games the votes of the voters in $N \backslash\{i\}$ are equally distributed in $[0,1]^{n-1}$, so that we have to use an $(n-1)$-fold integral. The counting of $(m, l)$-swings for the different possible shifts of the opinion of voter 1 can be condensed to a single expression: Given an ordered $j$-partition $S$, we denote by $\underline{S}$ the $j$-partition arising from $S$ by setting the vote of voters $i$ to alternative 1 . Similarly, we denote by $\bar{S}$ the $j$-partition arising from $S$ by setting the vote of voters $i$ to alternative $k$. Then $v(\underline{S})-v(\bar{S})$ counts the number of $(m, l)$-swings for voter $i$ given the preferences of the other voters in $N \backslash\{i\}$. By dividing by $k-1$ this number is contained in $[0,1]$. For continuous simple games the lowest possible alternative is 0 and the highest possible alternative is 1 , so that:

Definition 39. Let $v:[0,1]^{n} \rightarrow[0,1]$ be a continuous simple game. The (absolute) Banzhaf index $\mathrm{BZI}_{i}(v)$ of voter $i$ in $v$ is given by

$$
\begin{aligned}
& \int_{0}^{1} \ldots \int_{0}^{1}\left(v\left(x_{1}, \ldots, x_{i-1}, 1, x_{i+1}, \ldots, n\right)-v\left(x_{1}, \ldots, x_{i-1}, 0, x_{i+1}, \ldots, n\right)\right) \\
& \mathrm{d} x_{1} \ldots \mathrm{d} x_{i-1} \mathrm{~d} x_{i+1} \ldots \mathrm{d} x_{n}
\end{aligned}
$$

For the two continuous simple games, introduced at the beginning of this section, we obtain: 


$$
\begin{aligned}
& \operatorname{BZI}_{1}(\hat{v})=\int_{0}^{1} \int_{0}^{1}\left(\frac{1+2 x_{2}^{2}+3 x_{3}^{3}}{6}-\frac{0+2 x_{2}^{2}+3 x_{3}^{3}}{6}\right) \mathrm{d} x_{2} \mathrm{~d} x_{3}=\frac{1}{6} \\
& \mathrm{BZI}_{2}(\hat{v})=\int_{0}^{1} \int_{0}^{1}\left(\frac{1 x_{1}+2+3 x_{3}^{3}}{6}-\frac{1 x_{1}^{1}+0+3 x_{3}^{3}}{6}\right) \mathrm{d} x_{1} \mathrm{~d} x_{3}=\frac{2}{6} \\
& \mathrm{BZI}_{3}(\hat{v})=\int_{0}^{1} \int_{0}^{1}\left(\frac{1 x_{1}^{2}+2 x_{2}^{2}+3}{6}-\frac{1 x_{1}^{2}+2 x_{2}^{2}+0}{6}\right) \mathrm{d} x_{1} \mathrm{~d} x_{2}=\frac{3}{6}
\end{aligned}
$$

Since $\mathrm{BZI}_{1}(\hat{v})+\mathrm{BZI}_{2}(\hat{v})+\mathrm{BZI}_{3}(\hat{v})=1$ no normalization is necessary.

$$
\begin{aligned}
& \operatorname{BZI}_{1}(\tilde{v})=\int_{0}^{1} \int_{0}^{1}\left(x_{2}^{2} x_{3}^{3}-0\right) \mathrm{d} x_{2} \mathrm{~d} x_{3}=\frac{1}{12} \\
& \mathrm{BZI}_{2}(\tilde{v})=\int_{0}^{1} \int_{0}^{1}\left(x_{1} x_{3}^{3}-0\right) \mathrm{d} x_{1} \mathrm{~d} x_{3}=\frac{1}{8} \\
& \mathrm{BZI}_{3}(\tilde{v})=\int_{0}^{1} \int_{0}^{1}\left(x_{1} x_{2}^{2}-0\right) \mathrm{d} x_{1} \mathrm{~d} x_{2}=\frac{1}{6}
\end{aligned}
$$

After normalization we obtain $\frac{1}{9} \cdot(2,3,4)=(0 . \overline{2}, 0 . \overline{3}, 0 . \overline{4})$ for the (relative) Banzhaf index.

An axiomatization of the Banzhaf index for simple games was, e.g., given in [49]:

Lemma 15. Let $P: \mathcal{S}_{n} \rightarrow \mathbb{R}^{n}$ be a power index. If $P$ satisfies symmetry, the null voter property, the transfer axiom, and the Banzhaf total power, then P coincides with the Banzhaf index.

Definition 40. A power index $P: \mathcal{V}_{n} \rightarrow \mathbb{R}^{n}$ satisfies Banzhaf total power, if

$$
\sum_{i=1}^{n} \operatorname{BZI}_{i}(v)=\frac{1}{2^{n-1}} \cdot \sum_{i=1}^{n} \sum_{S \subseteq N \backslash\{i\}}(v(S \cup\{i\})-v(S))
$$

for all $v \in \mathcal{V}_{n}$, where $\mathcal{V}_{n}$ is a subclass of (binary) Boolean games.

Definition 40 can be restated directly for continuous Boolean games:

Definition 41. A power index $P: \mathbb{V}_{n} \rightarrow \mathbb{R}^{n}$ satisfies Banzhaf total power, if $\|\mathrm{BZI}(v)\|_{1}$ coincides with

$$
\begin{aligned}
& \sum_{i=1}^{n} \int_{0}^{1} \ldots \int_{0}^{1}\left(v\left(x_{1}, \ldots, x_{i-1}, 1, x_{i+1}, \ldots, n\right)-v\left(x_{1}, \ldots, x_{i-1}, 0, x_{i+1}, \ldots, n\right)\right) \\
& \mathrm{d} x_{1} \ldots \mathrm{d} x_{i-1} \mathrm{~d} x_{i+1} \ldots \mathrm{d} x_{n}
\end{aligned}
$$

for all $v \in \mathbb{V}_{n}$, where $\mathbb{V}_{n}$ is a subclass of continuous Boolean games.

Directly from the definitions we conclude:

Lemma 16. The Banzhaf index BZI is symmetric, positive, and satisfies the null voter property, the transfer axiom, and Banzhaf total power on $\mathbb{S}_{n}$.

Conjecture 3. Let $P: \mathbb{S}_{n} \rightarrow \mathbb{R}^{n}$ be a power index. If $P$ satisfies symmetry, the null voter property, the transfer axiom, and the Banzhaf total power, then P coincides with the Banzhaf index according to Definition 39. 


\subsection{Nucleolus}

Definition 42. Given a continuous simple game $v:[0,1]^{n} \rightarrow[0,1]$ and a vector $w \in[0,1]^{n}$ with $\|w\|_{1}=1$, the excess of a coalition $x \in[0,1]^{n}$ is given by $e^{v}(x, w)=v(x)-w^{T} x \in[-1,1]$.

The excess vector for the case of simple games is generalized to:

Definition 43. Given a continuous simple game $v:[0,1]^{n} \rightarrow[0,1]$ and a vector $w \in[0,1]^{n}$ with $\|w\|_{1}=1$, the excess function is given by $E_{w}^{v}:[-1,1] \rightarrow[0,1]$,

$$
c \mapsto \operatorname{vol}\left(\left\{x \in[0,1]^{n}: v(x)-w^{T} x \geq c\right\}\right)
$$

where $\operatorname{vol}(S)$ denotes the $n$-dimensional volume of a subset $S \subseteq \mathbb{R}^{n}$. (Here we assume that the mapping $v$ is regular enough, e.g., piecewise continuous, so that those volumes exist.)

Instead of the lexicographic ordering for two excess vectors we define:

Definition 44. For two integrable functions $f_{1}:[-1,1] \rightarrow[0,1]$ and $f_{2}:[-1,1] \rightarrow[0,1]$, we write $f_{1} \leq f_{2}$ if there exists a constant $c \in[-1,1]$ such that $f_{1}(y) \leq f_{2}(y)$ for all $y \in[c, 1]$ and $\int_{c}^{1} f_{1}(y) \mathrm{d} y<$ $\int_{c}^{1} f_{2}(y) \mathrm{d} y$.

Definition 45. For a continuous simple game $v:[0,1]^{n} \rightarrow[0,1]$ the nucleolus $\mathrm{Nuc}(\mathbf{v})$ is given by

$$
\left\{w \in[0,1]^{n}:\|w\|_{1}=1, E_{w}^{v} \leq E_{\hat{w}}^{v} \forall \hat{w} \in[0,1]^{n}:\|\hat{w}\|_{1}=1\right\}
$$

Conjecture 4. Under mild technical assumptions for a continuous simple game $v:[0,1]^{n} \rightarrow[0,1]$, we have $|\operatorname{Nuc}(v)| \leq 1$.

By definition the elements of the nucleolus are positive and efficient. Of course we also want to compute the nucleolus for our two examples. Unfortunately we have no general algorithm at hand, which is capable of solving the optimization problem stated in Definition 45. For $\hat{v}$ we can compute the nucleolus to be $\frac{1}{6} \cdot(1,2,3)$, i.e., it coincides with the Shapley-Shubik and the Banzhaf index, using a tailored analysis in Appendix C. For $\tilde{v}$ things seem to be much more complicated without the aid of theoretical results. For the similar two-voter example $v\left(x_{1}, x_{2}\right)=x_{1} x_{2}^{2}$ we compute numeric bounds for the elements in the nucleolus in Appendix C.

\section{Power Indices When Votes Are Not Equiprobable}

Both the Shapley-Shubik index and the Banzhaf index for simple games, $(j, k)$ simple games, or continuous simple games are based on the assumption that voters vote independently from each other and that they choose each alternative with equal probability. The first assumption is clearly violated in several practical contexts. Here we restrict ourselves to situations where this assumption is still met. An equal probability for all possible input alternatives makes a certain sense for (binary) simple games. Here one can have in mind that the roles of the alternatives are swapped if the proposal is logically negated. As argued in, e.g., [7], for the special case of $(3,2)$ simple games, where the central alternative is abstention, things are quite different. In some real-world legislatures, where abstention is allowed, the 
rate of abstention is rather low, while in others it is considerably higher. For the Banzhaf index different probabilities for the two options were, e.g., considered in [50].

For continuous simple games we model this more general situation by assuming a density function $f_{i}$, i.e., $f_{i}:[0,1] \rightarrow \mathbb{R}_{\geq 0}$ with $\int_{0}^{1} f_{i}(x) \mathrm{d} x=1$, for each voter $i \in N$. With this we propose:

Definition 46. Let $v:[0,1]^{n} \rightarrow[0,1]$ be a continuous simple game. The density Shapley-Shubik index $\operatorname{SSI}_{i}^{f}(v)$ of voter $i$ in $v$ is given by

$$
\begin{aligned}
& \frac{1}{n !} \cdot \sum_{\pi \in \mathcal{S}_{n}} \int_{0}^{1} \ldots \int_{0}^{1}\left(\left(v\left(\bar{\tau}\left(x, \pi^{-1}(i)-1\right)\right)-v\left(\bar{\tau}\left(x, \pi^{-1}(i)\right)\right)\right)\right. \\
& \left.+\left(v\left(\underline{\tau}\left(x, \pi^{-1}(i)\right)\right)-v\left(\underline{\tau}\left(x, \pi^{-1}(i)-1\right)\right)\right)\right) \cdot f_{1}\left(x_{1}\right) \ldots f_{n}\left(x_{n}\right) \mathrm{d} x_{1} \ldots \mathrm{d} x_{n}
\end{aligned}
$$

where $\mathcal{S}_{n}$ denotes the symmetric group on $n$ elements, i.e., the set of permutations or bijections from $\{1, \ldots, n\}$ to $\{1, \ldots, n\}$ and $f=\left(f_{1}, \ldots, f_{n}\right)$ is a vector of density functions.

For the special case of the weighted median aggregation rule this definition was (in its simplified version), e.g., used in [51]. As a small example we consider the median aggregation rule for a continuous simple game $v$ with three voters and density functions, which are given by

$$
\begin{aligned}
f_{1}(x) & =\frac{3}{4} \cdot\left(1-x^{2}\right) \\
f_{2}(x) & =\frac{3}{8} \cdot\left(1+x^{2}\right), \text { and } \\
f_{3}(x) & =\frac{3}{8} \cdot\left(1+x^{2}\right)
\end{aligned}
$$

for $x \in[-1,1]$ and zero otherwise. We can easily check that the three stated functions are indeed density functions. With this we have

$$
\begin{aligned}
\operatorname{SSI}_{1}^{f}(v) & =\int_{-1}^{1} \int_{-1}^{x_{1}} \int_{x_{1}}^{1} f(x) \mathrm{d} x_{2}, \mathrm{~d} x_{3} \mathrm{~d} x_{1}+\int_{-1}^{1} \int_{-1}^{x_{1}} \int_{x_{1}}^{1} f(x) \mathrm{d} x_{3}, \mathrm{~d} x_{2} \mathrm{~d} x_{1} \\
& =\frac{554}{13440} \approx 0.04122 \\
\operatorname{SSI}_{2}^{f}(v) & =\int_{-1}^{1} \int_{-1}^{x_{2}} \int_{x_{2}}^{1} f(x) \mathrm{d} x_{1}, \mathrm{~d} x_{3} \mathrm{~d} x_{2}+\int_{-1}^{1} \int_{-1}^{x_{2}} \int_{x_{2}}^{1} f(x) \mathrm{d} x_{3}, \mathrm{~d} x_{1} \mathrm{~d} x_{2} \\
& =\frac{563}{13440} \approx 0.04189 \\
\operatorname{SSI}_{3}^{f}(v) & =\int_{-1}^{1} \int_{-1}^{x_{3}} \int_{x_{3}}^{1} f(x) \mathrm{d} x_{1}, \mathrm{~d} x_{2} \mathrm{~d} x_{3}+\int_{-1}^{1} \int_{-1}^{x_{3}} \int_{x_{3}}^{1} f(x) \mathrm{d} x_{2}, \mathrm{~d} x_{1} \mathrm{~d} x_{3} \\
& =\frac{563}{13440} \approx 0.04189
\end{aligned}
$$

where we use the abbreviation $f(x)=f_{1}\left(x_{1}\right) \cdot f_{2}\left(x_{2}\right) \cdot f_{3}\left(x_{3}\right)$.

Definition 47. Let $v:[0,1]^{n} \rightarrow[0,1]$ be a continuous simple game. The density (absolute) Banzhaf index $\mathrm{BZI}_{i}^{f}(v)$ of voter $i$ in $v$ is given by

$$
\begin{aligned}
& \int_{0}^{1} \ldots \int_{0}^{1}\left(v\left(x_{1}, \ldots, x_{i-1}, 1, x_{i+1}, \ldots, n\right)-v\left(x_{1}, \ldots, x_{i-1}, 0, x_{i+1}, \ldots, n\right)\right) \\
& \cdot f_{1}\left(x_{1}\right) \ldots f_{i-1}\left(x_{i-1}\right) \cdot f_{i+1}\left(x_{i+1}\right) \ldots f_{n}\left(x_{n}\right) \mathrm{d} x_{1} \ldots \mathrm{d} x_{i-1} \mathrm{~d} x_{i+1} \ldots \mathrm{d} x_{n}
\end{aligned}
$$


where $f=\left(f_{1}, \ldots, f_{n}\right)$ is a vector of density functions.

\section{Conclusions}

Measurement of voting power is relevant in many practical applications. The widely used binary voting model does not fit for several economic problems like, e.g., tax rates or spending. Here we have proposed some definitions for continuous games and highlighted their similarity to the corresponding definitions for simple or $(j, k)$ simple games. Some first few assertions, known to be true for simple games, are proven to be valid for our new generalized definitions. We do not claim that we have found the ultimate truth, but want to stimulate the research for the right generalization by presenting our educated guess. It is a major task for the future, to transfer known results for simple games for continuous simple games and eventually modify our definitions if they do not seem to fit well for a majority of those results. The possibly weakest part of our suggestions are the generalizations of weightedness. However, here the situation even has not been resolved convincingly for $(j, k)$ simple games. A good benchmark for the proposed definitions of certain properties for simple, $(j, k)$ simple, and continuous simple games would be, if the version for simple games arises as a specialization to $(2,2)$ simple games, and the version for continuous simple games arises by taking the limit $j, k \rightarrow \infty$.

For three power indices from cooperative game theory we have proposed generalizations for continuous simple games and started to study their properties. A litmus test might be to check whether those defined power indices can be axiomatized in a similar fashion than their binary counterparts. For the Shapley-Shubik and the Banzhaf index we have conjectured such axiomatizations. The key to a possible proof of those conjectures might be a generalized definition of unanimity games.

We have illustrated our generalized power indices by computing the respective values for several examples. For some parameterized classes of such examples it is indeed possible to write down easy formulas, which will be delayed to a more technical follow-up paper. For the proposed generalization of the nucleolus even an algorithmic way to compute the corresponding set is missing. Maybe it also makes sense to consider generalizations for other of the known power indices for simple games.

We really hope that this paper can partially contribute to the development of a unified framework for measuring decision power and stimulates further research in that direction.

\section{Conflicts of Interest}

The author declares no conflict of interest.

\section{References}

1. Napel, S.; Widgrén, M. Power measurement as sensitivity analysis a unified approach. J. Theor. Polit. 2004, 16, 517-538.

2. Felsenthal, D.S.; Machover, M. Myths and meanings of voting power comments on a symposium. J. Theor. Polit. 2001, 13, 81-97.

3. Steunenberg, B.; Schmidtchen, D.; Koboldt, C. Strategic power in the European Union evaluating the distribution of power in policy games. J. Theor. Polit. 1999, 11, 339-366. 
4. Holler, M.J.; Nurmi, H. Reflections on power, voting, and voting power. In Power, Voting, and Voting Power: 30 Years After; Springer: Berlin/Heidelberg, Germany, 2013; pp. 1-24.

5. Le Breton, M.; Montero, M.; Zaporozhets, V. Voting power in the EU Council of Ministers and fair decision making in distributive politics. Math. Soc. Sci. 2012, 63, 159-173.

6. Taylor, A.D.; Zwicker, W.S. Simple Games. Desirability Relations, Trading, Pseudoweightings; Princeton University Press: Princeton, NJ, USA, 1999; p. 246.

7. Felsenthal, D.S.; Machover, M. Ternary voting games. Int. J. Game Theory 1997, 26, 335-351.

8. Freixas, J.; Zwicker, W.S. Weighted voting, abstention, and multiple levels of approval. Soc. Choice Welf. 2003, 21, 399-431.

9. Bolger, E.M. The Banzhaf index for multicandidate presidential elections. SIAM J. Algebr. Discret. Methods 1983, 4, 442-458.

10. Bolger, E.M. Power indices for multicandidate voting games. Int. J. Game Theory 1986, 15, 175-186.

11. Bolger, E.M. A characterization of an extension of the Banzhaf value to multicandidate voting games. SIAM J. Discret. Math. 1990, 3, 466-477.

12. Bolger, E.M. A value for games with $n$ players and $r$ alternatives. Int. J. Game Theory 1993, 22, 319-334.

13. Bolger, E.M. A consistent value for games with $n$ players and $r$ alternatives. Int. J. Game Theory 2000, 29, 93-99.

14. Bolger, E.M. Characterizations of two power indices for voting games with $r$ alternatives. Soc. Choice Welf. 2002, 19, 709-721.

15. Freixas, J. Banzhaf measures for games with several levels of approval in the input and output. Ann. Oper. Res. 2005, 137, 45-66.

16. Freixas, J. The Shapley-Shubik power index for games with several levels of approval in the input and output. Decis. Support Syst. 2005, 39, 185-195.

17. DeMarzo, P.M.; Vayanos, D.; Zwiebel, J. Persuasion bias, social influence, and unidimensional opinions. Q. J. Econ. 2003, 118, 909-968.

18. Grabisch, M.; Rusinowska, A. A model of influence with a continuum of actions. J. Math. Econ. 2011, 47, 576-587.

19. Maaser, N.; Napel, S. Equal representation in two-tier voting systems. Soc. Choice Welf. 2007, 28, 401-420.

20. Maaser, N.; Napel, S. The Mean Voter, the Median Voter, and Welfaremaximizing Voting Weights; Technical Report, Discussion Paper 59; Public Choice Research Centre, University of Turku: Turku, Finland, 2012.

21. Maaser, N.; Napel, S. A note on the direct democracy deficit in two-tier voting. Math. Soc. Sci. 2012, 63, 174-180.

22. Isbell, J.R. A class of simple games. Duke Math. J. 1958, 25, 423-439.

23. Kurz, S. On minimum sum representations for weighted voting games. Ann. Oper. Res. 2012, 196, 361-369.

24. Kurz, S.; Tautenhahn, N. On Dedekind's problem for complete simple games. Int. J. Game Theory 2013, 42, 411-437. 
25. Bertini, C.; Freixas, J.; Gambarelli, G.; Stach, I. Comparing power indices. Int. Game Theory Rev. 2013, 15, 1340004:1-1340004:19.

26. Shapley, L.S.; Shubik, M. A method for evaluating the distribution of power in a committee system. Am. Polit. Sci. Rev. 1954, 48, 787-792.

27. Banzhaf, J.F. Weighted voting doesn't work: A mathematical analysis. Rutgers Law Rev. 1965, 19, 317-343.

28. Schmeidler, D. The nucleolus of a characteristic function game. SIAM J. Appl. Math. 1969, 17, 1163-1170.

29. Montero, M. Noncooperative foundations of the nucleolus in majority games. Games Econ. Behav. 2006, 54, 380-397.

30. Montero, M. On the nucleolus as a power index. In Power, Voting, and Voting Power: 30 Years After; Springer: Berlin/Heidelberg, Germany, 2013; pp. 283-299.

31. Felsenthal, D.S.; Machover, M. Models and Reality: The curious case of the absent abstention. In Power, Voting, and Voting Power: 30 Years After; Springer: Berlin/Heidelberg, Germany, 2013; pp. 73-86.

32. Freixas, J. Probabilistic power indices for voting rules with abstention. Math. Soc. Sci. 2012, 64, 89-99.

33. Downs, A. An Economic Theory of Democracy; Harper Collins: New York, NY, USA, 1957.

34. Downs, A. An economic theory of political action in a democracy. J. Polit. Econ. 1957, 65, 135-150.

35. Meltzer, A.H.; Richard, S.F. A rational theory of the size of government. J. Polit. Econ. 1981, 89, 914-927.

36. Romer, T. Individual welfare, majority voting, and the properties of a linear income tax. J. Public Econ. 1975, 4, 163-185.

37. Stadelmann, D.; Portmann, M.; Eichenberger, R. Evaluating the median voter model's explanatory power. Econ. Lett. 2012, 114, 312-314.

38. Lehrer, K.; Wagner, C. Rational Consensus in Science and Society: A Philosophical and Mathematical Study; Springer: Berlin/Heidelberg, Germany, 1981; Volume 21.

39. Hegselmann, R.; Krause, U. Opinion dynamics and bounded confidence models, analysis, and simulation. Available online: http://jasss.soc.surrey.ac.uk/5/3/2.html (accessed on 20 December 2013).

40. Katz, E.; Lazarsfeld, P.F. Personal Influence: The Part Played by People in the Flow of Mass Communications, 2nd ed.; Transaction Publishers: New Brunswick, NJ, USA, 2009.

41. Van Den Brink, R.; Rusinowska, A.; Steffen, F. Measuring power and satisfaction in societies with opinion leaders: An axiomatization. Soc. Choice Welf. 2013, 41, 671-683.

42. Grabisch, M.; Rusinowska, A. Different approaches to influence based on social networks and simple games. In Collective Decision Making; Springer: Berlin/Heidelberg, Germany, 2010; pp. 185-209.

43. Grabisch, M.; Rusinowska, A. A model of influence with an ordered set of possible actions. Theory Decis. 2010, 69, 635-656. 
44. Grabisch, M.; Rusinowska, A. Influence functions, followers and command games. Games Econ. Behav. 2011, 72, 123-138.

45. Maruani, E.; Grabisch, M.; Rusinowska, A. A study of the dynamic of influence through differential equations. RAIRO-Oper. Res. 2012, 46, 83-106.

46. Hoede, C.; Bakker, R.R. A theory of decisional power. J. Math. Sociol. 1982, 8, 309-322.

47. Jackson, M.O. Social and Economic Networks; Princeton University Press: Princeton, NJ, USA, 2010.

48. Dubey, P. On the uniqueness of the shapley value. Int. J. Game Theory 1975, 4, 131-139.

49. Dubey, P.; Shapley, L.S. Mathematical properties of the Banzhaf power index. Math. Oper. Res. 1979, 4, 99-131.

50. Kaniovski, S. The exact bias of the Banzhaf measure of power when votes are neither equiprobable nor independent. Soc. Choice Welf. 2008, 31, 281-300.

51. Kurz, S.; Maaser, N.; Napel, S. On the egalitarian weights of nations. 2013, submitted for publication.

\section{A. Determining the SSI for Two Continuous Simple Games}

For the two continuous simple games $\hat{v}$ and $\tilde{v}$ from Section 6 we compute the Shapley-Shubik indices. In Tables A1-A3 we give the respective summands for each permutation $\pi \in \mathcal{S}_{3}$ for $\hat{v}$. Summarizing the results we obtain

$$
\operatorname{SSI}(\hat{v})=\left(\frac{1}{6}, \frac{2}{6}, \frac{3}{6}\right)
$$

Table A1. $\mathrm{SSI}_{1}(\hat{v})$ for $\hat{v}\left(x_{1}, x_{2}, x_{3}\right)=\frac{1}{6} \cdot\left(1 x_{1}^{2}+2 x_{2}^{2}+3 x_{3}^{2}\right)$.

\begin{tabular}{lc}
\hline$\pi \in \mathcal{S}_{3}$ & 3 -fold integral \\
\hline$(1,2,3)$ & $\int_{x \in[0,1]^{3}}\left(\frac{6}{6}-\frac{x_{1}^{2}+5}{6}+\frac{x_{1}^{2}}{6}-\frac{0}{6}\right) \mathrm{d} x=\frac{1}{6}$ \\
$(1,3,2)$ & $\int_{x \in[0,1]^{3}}\left(\frac{6}{6}-\frac{x_{1}^{2}+5}{6}+\frac{x_{1}^{2}}{6}-\frac{0}{6}\right) \mathrm{d} x=\frac{1}{6}$ \\
$(2,1,3)$ & $\int_{(2,3,1)}\left(\frac{2 x_{2}^{2}+4}{6}-\frac{x_{1}^{2}+2 x_{2}^{2}+3}{6}+\frac{x_{1}^{2}+2 x_{2}^{2}}{6}-\frac{2 x_{2}^{2}}{6}\right) \mathrm{d} x=\frac{1}{6}$ \\
$(3,1,2)$ & $\int_{x \in[0,1]^{3}}\left(\frac{2 x_{2}^{2}+3 x_{3}^{2}+1}{6}-\frac{x_{1}^{2}+2 x_{2}^{2}+3 x_{3}^{2}}{6}+\frac{x_{1}^{2}+2 x_{2}^{2}+3 x_{3}^{2}}{6}-\frac{2 x_{2}^{2}+3 x_{3}^{2}}{6}\right) \mathrm{d} x=\frac{1}{6}$ \\
$(3,2,1)$ & $\int_{x \in[0,1]^{3}}\left(\frac{2 x_{2}^{2}+3 x_{3}^{2}+1}{6}-\frac{x_{1}^{2}+2 x_{2}^{2}+3 x_{3}^{2}}{6}+\frac{x_{1}^{2}+2 x_{2}^{2}+3 x_{3}^{2}}{6}-\frac{2 x_{2}^{2}+3 x_{3}^{2}}{6}\right) \mathrm{d} x=\frac{1}{6}$ \\
\hline
\end{tabular}

In Tables A4-A6 we give the respective summands for each permutation $\pi \in \mathcal{S}_{3}$ for $\tilde{v}$. Summarizing the results we obtain

$$
\operatorname{SSI}(\tilde{v})=\left(\frac{35}{144}, \frac{50}{144}, \frac{59}{144}\right)=(0.2430 \overline{5}, 0.347 \overline{2}, 0.4097 \overline{2})
$$


Table A2. $\mathrm{SSI}_{2}(\hat{v})$ for $\hat{v}\left(x_{1}, x_{2}, x_{3}\right)=\frac{1}{6} \cdot\left(1 x_{1}^{2}+2 x_{2}^{2}+3 x_{3}^{2}\right)$.

\begin{tabular}{cc}
\hline$\pi \in \mathcal{S}_{3}$ & 3 -fold integral \\
\hline$(2,1,3)$ & $\int_{x \in[0,1]^{3}}\left(\frac{6}{6}-\frac{x_{2}^{2}+4}{6}+\frac{x_{2}^{2}}{6}-\frac{0}{6}\right) \mathrm{d} x=\frac{2}{6}$ \\
$(2,3,1)$ & $\int_{x \in[0,1]^{3}}\left(\frac{6}{6}-\frac{x_{2}^{2}+4}{6}+\frac{x_{2}^{2}}{6}-\frac{0}{6}\right) \mathrm{d} x=\frac{2}{6}$ \\
$(1,2,3)$ & $\int_{x \in[0,1]^{3}}\left(\frac{x_{1}^{2}+5}{6}-\frac{x_{1}^{2}+2 x_{2}^{2}+3}{6}+\frac{x_{1}^{2}+2 x_{2}^{2}}{6}-\frac{x_{1}^{2}}{6}\right) \mathrm{d} x=\frac{2}{6}$ \\
$(3,2,1)$ & $\int_{x \in[0,1]^{3}}\left(\frac{3 x_{3}^{2}+3}{6}-\frac{2 x_{2}^{2}+3 x_{3}^{2}+1}{6}+\frac{2 x_{2}^{2}+3 x_{3}^{2}}{6}-\frac{3 x_{3}^{2}}{6}\right) \mathrm{d} x=\frac{2}{6}$ \\
$(1,3,2)$ & $\int_{x \in[0,1]^{3}}\left(\frac{x_{1}^{2}+3 x_{3}^{2}+2}{6}-\frac{x_{1}^{2}+2 x_{2}^{2}+3 x_{3}^{2}}{6}+\frac{x_{1}^{2}+2 x_{2}^{2}+3 x_{3}^{2}}{6}-\frac{x_{1}^{2}+3 x_{3}^{2}}{6}\right) \mathrm{d} x=\frac{2}{6}$ \\
$(3,1,2)$ & $\int_{x \in[0,1]^{3}}\left(\frac{x_{1}^{2}+3 x_{3}^{2}+2}{6}-\frac{x_{1}^{2}+2 x_{2}^{2}+3 x_{3}^{2}}{6}+\frac{x_{1}^{2}+2 x_{2}^{2}+3 x_{3}^{2}}{6}-\frac{x_{1}^{2}+3 x_{3}^{2}}{6}\right) \mathrm{d} x=\frac{2}{6}$ \\
\hline
\end{tabular}

Table A3. $\mathrm{SSI}_{3}(\hat{v})$ for $\hat{v}\left(x_{1}, x_{2}, x_{3}\right)=\frac{1}{6} \cdot\left(1 x_{1}^{2}+2 x_{2}^{2}+3 x_{3}^{2}\right)$.

\begin{tabular}{lc}
\hline$\pi \in \mathcal{S}_{3}$ & 3 -fold integral \\
\hline$(3,1,2)$ & $\int_{x \in[0,1]^{3}}\left(\frac{6}{6}-\frac{3 x_{3}^{2}+3}{6}+\frac{3 x_{3}^{2}}{6}-\frac{0}{6}\right) \mathrm{d} x=\frac{3}{6}$ \\
$(3,2,1)$ & $\int_{x \in[0,1]^{3}}\left(\frac{6}{6}-\frac{3 x_{3}^{2}+3}{6}+\frac{3 x_{3}^{2}}{6}-\frac{0}{6}\right) \mathrm{d} x=\frac{3}{6}$ \\
$(1,3,2)$ & $\int_{(2,3,1)}\left(\frac{x_{1}^{2}+5}{6}-\frac{x_{1}^{2}+3 x_{3}^{2}+2}{6}+\frac{x_{1}^{2}+3 x_{3}^{2}}{6}-\frac{x_{1}^{2}}{6}\right) \mathrm{d} x=\frac{3}{6}$ \\
$(1,2,3)$ & $\int_{x \in[0,1]^{3}}\left(\frac{2 x_{2}^{2}+4}{6}-\frac{2 x_{2}^{2}+3 x_{3}^{2}+1}{6}+\frac{2 x_{2}^{2}+3 x_{3}^{2}}{6}-\frac{2 x_{2}^{2}}{6}\right) \mathrm{d} x=\frac{3}{6}$ \\
$(2,1,3)$ & $\int_{x \in[0,1]^{3}}\left(\frac{x_{1}^{2}+2 x_{2}^{2}+3}{6}-\frac{x_{1}^{2}+2 x_{2}^{2}+3 x_{3}^{2}}{6}+\frac{x_{1}^{2}+2 x_{2}^{2}+3 x_{3}^{2}}{6}-\frac{x_{1}^{2}+2 x_{2}^{2}}{6}\right) \mathrm{d} x=\frac{3}{6}$ \\
& $\int_{x \in[0,1]^{3}}\left(\frac{x_{1}^{2}+2 x_{2}^{2}+3}{6}-\frac{x_{1}^{2}+2 x_{2}^{2}+3 x_{3}^{2}}{6}+\frac{x_{1}^{2}+2 x_{2}^{2}+3 x_{3}^{2}}{6}-\frac{x_{1}^{2}+2 x_{2}^{2}}{6}\right) \mathrm{d} x=\frac{3}{6}$ \\
\hline
\end{tabular}

Table A4. $\operatorname{SSI}_{1}(\tilde{v})$ for $\tilde{v}\left(x_{1}, x_{2}, x_{3}\right)=x_{1} x_{2}^{2} x_{3}^{3}$.

\begin{tabular}{lc}
\hline$\pi \in \mathcal{S}_{3}$ & 3 -fold integral \\
\hline$(1,2,3)$ & $\int_{(1,3,2)}\left(1-x_{1}+0-0\right) \mathrm{d} x=\frac{1}{2}$ \\
$(2,1,3)$ & $\int_{x \in[0,1]^{3}}\left(1-x_{1}+0-0\right) \mathrm{d} x=\frac{1}{2}$ \\
$(2,3,1)$ & $\int_{x \in[0,1]^{3}}\left(x_{2}^{2}-x_{1} x_{2}^{2}+0-0\right) \mathrm{d} x=\frac{1}{6}$ \\
$(3,1,2)$ & $\int_{x \in[0,1]^{3}}\left(x_{2}^{2} x_{3}^{3}-x_{1} x_{2}^{2} x_{3}^{3}+x_{1} x_{2}^{2} x_{3}^{3}-0\right) \mathrm{d} x=\frac{1}{12}$ \\
$(3,2,1)$ & $\int_{x \in[0,1]^{3}}\left(x_{2}^{2} x_{3}^{3}-x_{1} x_{2}^{2} x_{3}^{3}+x_{1} x_{2}^{2} x_{3}^{3}-0\right) \mathrm{d} x=\frac{1}{12}$ \\
\hline
\end{tabular}


Table A5. $\mathrm{SSI}_{2}(\tilde{v})$ for $\tilde{v}\left(x_{1}, x_{2}, x_{3}\right)=x_{1} x_{2}^{2} x_{3}^{3}$.

\begin{tabular}{cc}
\hline$\pi \in \mathcal{S}_{3}$ & 3 -fold integral \\
\hline$(1,2,3)$ & $\int_{x \in[0,1]^{3}}\left(x_{1}-x_{1} x_{2}^{2}+0-0\right) \mathrm{d} x=\frac{1}{3}$ \\
$(1,3,2)$ & $\int_{x \in[0,1]^{3}}\left(x_{1} x_{3}^{3}-x_{1} x_{2}^{2} x_{3}^{3}+x_{1} x_{2}^{2} x_{3}^{3}-0\right) \mathrm{d} x=\frac{1}{8}$ \\
$(2,1,3)$ & $\int_{x \in[0,1]^{3}}\left(1-x_{2}^{2}+0-0\right) \mathrm{d} x=\frac{2}{3}$ \\
$(2,3,1)$ & $\int_{(3,1,2)}\left(1-x_{2}^{2}+0-0\right) \mathrm{d} x=\frac{2}{3}$ \\
$(3,2,1)$ & $\int_{x \in[0,1]^{3}}\left(x_{1} x_{3}^{3}-x_{1} x_{2}^{2} x_{3}^{3}+x_{1} x_{2}^{2} x_{3}^{3}-0\right) \mathrm{d} x=\frac{1}{8}$ \\
$\int_{x \in[0,1]^{3}}\left(x_{3}^{3}-x_{2}^{2} x_{3}^{3}+0-0\right) \mathrm{d} x=\frac{1}{6}$ \\
\hline
\end{tabular}

Table A6. $\mathrm{SSI}_{3}(\tilde{v})$ for $\tilde{v}\left(x_{1}, x_{2}, x_{3}\right)=x_{1} x_{2}^{2} x_{3}^{3}$.

\begin{tabular}{cc}
\hline$\pi \in \mathcal{S}_{3}$ & 3 -fold integral \\
\hline$(1,2,3)$ & $\int_{x \in[0,1]^{3}}\left(x_{1} x_{2}^{2}-x_{1} x_{2}^{2} x_{3}^{3}+x_{1} x_{2}^{2} x_{3}^{3}-0\right) \mathrm{d} x=\frac{1}{6}$ \\
$(1,3,2)$ & $\int_{x \in[0,1]^{3}}\left(x_{1}-x_{1} x_{3}^{3}+0-0\right) \mathrm{d} x=\frac{3}{8}$ \\
$(2,1,3)$ & $\int_{x \in[0,1]^{3}}\left(x_{1} x_{2}^{2}-x_{1} x_{2}^{2} x_{3}^{3}+x_{1} x_{2}^{2} x_{3}^{3}-0\right) \mathrm{d} x=\frac{1}{6}$ \\
$(2,3,1)$ & $\int_{x \in[0,1]^{3}}\left(x_{2}^{2}-x_{2}^{2} x_{3}^{3}+0-0\right) \mathrm{d} x=\frac{1}{4}$ \\
$(3,1,2)$ & $\int_{x \in[0,1]^{3}}\left(1-x_{3}^{3}+0-0\right) \mathrm{d} x=\frac{3}{4}$ \\
$(3,2,1)$ & $\int_{x \in[0,1]^{3}}\left(1-x_{3}^{3}+0-0\right) \mathrm{d} x=\frac{3}{4}$ \\
\hline
\end{tabular}

\section{B. Determining the SSI for a Weighted Median Aggregation Rule}

In order to illustrate Lemma 14 we compute the Shapley-Shubik index of a continuous simple voting game, which is given as the weighted median. We consider a case of 4 voters with weights $5,3,2,1$. The situation may be described as weighted binary game $[6 ; 5,3,2,1]=[3 ; 2,1,1,1]$, so that we may also consider the weights $2,1,1$, and 1 . Since no subset of the weights sums to exactly half of the weight sum the median is uniquely determined in every case. One can easily compute that $\operatorname{SSI}([3 ; 2,1,1,1])=$ $\left(\frac{1}{2}, \frac{1}{6}, \frac{1}{6}, \frac{1}{6}\right)$. In the subsequent subsections we will compute the Shapley-Shubik index directly using Definition 35, which is indeed a somewhat lengthy computation. 


\section{B.1. Shapley-Shubik Power for Voter 2}

2.1.1. $\pi \in\{(2,1,3,4),(2,1,4,3),(2,3,1,4),(2,3,4,1),(2,4,1,3),(2,4,3,1)\}$

For permutations $\pi$, where $\pi(1)=2$, we have $v\left(\bar{\tau}\left(x, \pi^{-1}(2)-1\right)\right)=1, v\left(\bar{\tau}\left(x, \pi^{-1}(2)\right)\right)=1$, $v\left(\underline{\tau}\left(x, \pi^{-1}(2)\right)\right)=0$, and $v\left(\underline{\tau}\left(x, \pi^{-1}(2)-1\right)\right)=0$, so that the value of the 4-fold integral is given by 0 .

2.1.2. $\pi \in\{(3,2,1,4),(3,2,4,1),(4,2,1,3),(4,2,3,1)\}$

For permutations $\pi$, where $\pi(2)=2$ and $\pi(1) \neq 1$, we have $v\left(\bar{\tau}\left(x, \pi^{-1}(2)-1\right)\right)=1$, $v\left(\bar{\tau}\left(x, \pi^{-1}(2)\right)\right)=1, v\left(\underline{\tau}\left(x, \pi^{-1}(2)\right)\right)=0$, and $v\left(\underline{\tau}\left(x, \pi^{-1}(2)-1\right)\right)=0$, so that the value of the 4 -fold integral is given by 0 .

2.1.3. $\pi=(1,2,3,4), \pi=(1,2,4,3)$

For the permutations $\pi=(1,2,3,4)$ and $\pi=(1,2,4,3)$ we have $v\left(\bar{\tau}\left(x, \pi^{-1}(2)-1\right)\right)=1$ and $v\left(\underline{\tau}\left(x, \pi^{-1}(2)-1\right)\right)=0$. If $x_{1} \leq x_{2}$ then $v\left(\bar{\tau}\left(x, \pi^{-1}(2)\right)\right)=x_{2}$ and $v\left(\underline{\tau}\left(x, \pi^{-1}(2)\right)\right)=x_{1}$. Similarly, if $\mathrm{I} x_{1} \geq x_{2}$ then $v\left(\bar{\tau}\left(x, \pi^{-1}(2)\right)\right)=x_{1}$ and $v\left(\underline{\tau}\left(x, \pi^{-1}(2)\right)\right)=x_{2}$. Thus for these two permutations the value of the 4 -fold integral is given by

$$
\begin{aligned}
& \int_{0}^{1}\left(1-\left(\int_{0}^{x_{2}} x_{2} \mathrm{~d} x_{1}+\int_{x_{2}}^{1} x_{1} \mathrm{~d} x_{1}\right)+\left(\int_{0}^{x_{2}} x_{1} \mathrm{~d} x_{1}+\int_{x_{2}}^{1} x_{2} \mathrm{~d} x_{1}\right)-0\right) \mathrm{d} x_{2} \\
= & \int_{0}^{1} \frac{1}{2}+x_{2}\left(1-x_{2}\right) \mathrm{d} x_{2}=\frac{2}{3}
\end{aligned}
$$

2.1.4. $\pi \in\{(1,3,2,4),(1,4,2,3),(3,1,2,4),(4,1,2,3)\}$

For the permutation $\pi=(1,3,2,4)$ we consider the six different strict orderings of $x_{1}, x_{2}$, and $x_{3}$ in Table A7. The respective integrals over $x_{1}, x_{3}$, and $x_{4}$ are stated in Table A8.

Table A7. Values of $v$ for $\pi=(1,3,2,4)$.

\begin{tabular}{lcccc}
\hline Ordering $v\left(\bar{\tau}\left(x, \pi^{-1}(2)-1\right)\right) v\left(\bar{\tau}\left(x, \pi^{-1}(2)\right)\right) v\left(\underline{\tau}\left(x, \pi^{-1}(2)\right)\right) v\left(\underline{\tau}\left(x, \pi^{-1}(2)-1\right)\right)$ \\
\hline$x_{1}<x_{2}<x_{3}$ & $x_{3}$ & $x_{2}$ & $x_{1}$ & $x_{1}$ \\
$x_{1}<x_{3}<x_{2}$ & $x_{3}$ & $x_{3}$ & $x_{1}$ & $x_{1}$ \\
$x_{2}<x_{1}<x_{3}$ & $x_{3}$ & $x_{1}$ & $x_{1}$ & $x_{1}$ \\
$x_{2}<x_{3}<x_{1}$ & $x_{1}$ & $x_{1}$ & $x_{3}$ & $x_{3}$ \\
$x_{3}<x_{1}<x_{2}$ & $x_{1}$ & $x_{1}$ & $x_{1}$ & $x_{3}$ \\
$x_{3}<x_{2}<x_{1}$ & $x_{1}$ & $x_{1}$ & $x_{2}$ & $x_{3}$ \\
\hline
\end{tabular}

Summing the right hand sides of the rows of Table A8 and integrating over $x_{2} \in[0,1]$ yields

$$
\int_{0}^{1} \frac{1}{6} \mathrm{~d} x_{2}=\frac{1}{6} .
$$

Due to symmetry we obtain the same value for $\pi=(1,4,2,3), \pi=(3,1,2,4)$, and $\pi=(4,1,2,3)$. 
Table A8. Auxiliary integrals for $\pi=(1,3,2,4)$.

\begin{tabular}{ll}
\hline Ordering & $\int_{\left(x_{1}, x_{3}, x_{4}\right)} \star$ \\
\hline$x_{1}<x_{2}<x_{3}$ & $\int_{0}^{x_{2}} \int_{x_{2}}^{1}\left(x_{3}-x_{2}+x_{1}-x_{1}\right) \mathrm{d} x_{3} \mathrm{~d} x_{1}=\frac{x_{2}^{3}-2 x_{2}^{2}+x_{2}}{2}$ \\
$x_{1}<x_{3}<x_{2}$ & $\int_{0}^{x_{2}} \int_{x_{1}}^{x_{2}}\left(x_{3}-x_{3}+x_{1}-x_{1}\right) \mathrm{d} x_{3} \mathrm{~d} x_{1}=0$ \\
$x_{2}<x_{1}<x_{3}$ & $\int_{x_{2}}^{1} \int_{x_{1}}^{1}\left(x_{3}-x_{1}+x_{1}-x_{1}\right) \mathrm{d} x_{3} \mathrm{~d} x_{1}=\frac{-x_{2}^{3}+3 x_{2}^{2}-3 x_{2}+1}{6}$ \\
$x_{2}<x_{3}<x_{1}$ & $\int_{x_{2}}^{1} \int_{x_{3}}^{1}\left(x_{1}-x_{1}+x_{3}-x_{3}\right) \mathrm{d} x_{1} \mathrm{~d} x_{3}=0$ \\
$x_{3}<x_{1}<x_{2}$ & $\int_{0}^{x_{2}} \int_{x_{3}}^{x_{2}}\left(x_{1}-x_{1}+x_{1}-x_{3}\right) \mathrm{d} x_{1} \mathrm{~d} x_{3}=\frac{x_{2}^{3}}{6}$ \\
$x_{3}<x_{2}<x_{1}$ & $\int_{0}^{x_{2}} \int_{x_{2}}^{1}\left(x_{1}-x_{1}+x_{2}-x_{3}\right) \mathrm{d} x_{1} \mathrm{~d} x_{3}=\frac{x_{2}^{2}-x_{2}^{3}}{2}$ \\
\hline
\end{tabular}

2.1.5. $\pi=(3,4,2,1), \pi=(4,3,2,1)$

For the permutations $\pi=(3,4,2,1)$ and $\pi=(4,3,2,1)$ we have $v\left(\bar{\tau}\left(x, \pi^{-1}(2)-1\right)\right)=1$, $v\left(\bar{\tau}\left(x, \pi^{-1}(2)-1\right)\right)=\max \left(x_{2}, x_{3}, x_{4}\right), v\left(\underline{\tau}\left(x, \pi^{-1}(2)-1\right)\right)=0$, and $v\left(\underline{\tau}\left(x, \pi^{-1}(2)\right)\right)=$ $\min \left(x_{2}, x_{3}, x_{4}\right)$. Thus for these two permutations the value of the 4 -fold integral is given by

$$
\int_{0}^{1} \int_{0}^{1} \int_{0}^{1}\left(1-\max \left(x_{2}, x_{3}, x_{4}\right)+\min \left(x_{2}, x_{3}, x_{4}\right)\right) \mathrm{d} x_{2} \mathrm{~d} x_{3} \mathrm{~d} x_{4}=\frac{1}{2}
$$

2.1.6. $\pi \in\{(1,3,4,2),(1,4,3,2),(3,1,4,2),(4,1,3,2),(3,4,1,2),(4,3,1,2)\}$

For permutations $\pi$ with $\pi(4)=2$, we consider the three cases whether the maximum and minimum of $x_{1}, x_{3}, x_{4}$ is equal to $x_{1}$ or another number. The respective values of $v$ are stated in Table A9, where we use the abbreviation $x^{\prime}=\left(x_{1}, x_{3}, x_{4}\right)$.

Table A9. Values of $v$ for permutations $\pi$ with $\pi(4)=2$.

\begin{tabular}{cccc}
\hline $\max x^{\prime}$ & $\min x^{\prime}$ & $v\left(\bar{\tau}\left(x, \pi^{-1}(2)-1\right)\right)$ & $v\left(\bar{\tau}\left(x, \pi^{-1}(2)\right)\right)$ \\
\hline$x_{1}$ & $\neq x_{1}$ & $x_{1}$ & $\left\{\begin{array}{r}\max \left(x_{2}, x_{3}, x_{4}\right): x_{2} \leq x_{1} \\
x_{1}: x_{2} \geq x_{1}\end{array}\right.$ \\
$\neq x_{1}$ & $x_{1}$ & $\min \left(x_{3}, x_{4}\right)$ & $x_{1}$ \\
$\neq x_{1}$ & $\neq x_{1}$ & $v\left(\underline{\tau}\left(x, \pi^{-1}(2)\right)\right)$ & $v\left(\underline{\tau}\left(x, x^{-1}(2)-1\right)\right)$ \\
\hline $\max x^{\prime}$ & $\min x^{\prime}$ & $\max \left(x_{2}, x_{3}, x_{4}\right)$ & $\max \left(x_{3}, x_{4}\right)$ \\
\hline$x_{1}$ & $\neq x_{1}$ & $x_{1}$ & $\left\{\begin{array}{r}\min \left(x_{2}, x_{3}, x_{4}\right): x_{2} \geq x_{1} \\
x_{1}: x_{2} \leq x_{1}\end{array}\right.$ \\
$\neq x_{1}$ & $x_{1}$ & $x_{1}$ \\
$\neq x_{1}$ & $\neq x_{1}$ & & $x_{1}$ \\
\hline
\end{tabular}

By looking at the 24 strict orderings of $x_{1}, x_{2}, x_{3}, x_{4}$ we compute the 4 -fold to be $\frac{1}{6}$. (For 4 orderings we obtain a value of $\frac{1}{60}$, for 12 orderings a value of $\frac{1}{120}$, and for 8 orderings a value of 0 .) 
2.1.7. Summarizing the 24 Permutations for $\operatorname{SSI}_{2}(v)$

$$
\operatorname{SSI}_{2}(v)=\frac{1}{4 !} \cdot\left(6 \cdot 0+4 \cdot 0+2 \cdot \frac{2}{3}+4 \cdot \frac{1}{6}+2 \cdot \frac{1}{2}+6 \cdot \frac{1}{6}\right)=\frac{4}{24}=\frac{1}{6}
$$

\section{B.2. Shapley-Shubik Power for Voter 3 and Voter 4}

Since voters 3 and 4 behave as voter 2 , i.e., they are symmetric, in the weighted median, we obtain $\operatorname{SSI}_{3}(v)=\operatorname{SSI}_{4}(v)=\frac{1}{6}$.

\section{B.3. Shapley-Shubik Power for Voter 1}

Assuming Conjecture 1 we would obtain

$$
\operatorname{SSI}_{1}(v)=1-\operatorname{SSI}_{2}(v)-\operatorname{SSI}_{3}(v)-\operatorname{SSI}_{4}(v)=\frac{1}{2}
$$

By an computation analogue to Subsection B.1 we can confirm this value directly. For completeness we give the entire calculation below.

2.3.1. $\pi \in\{(1,2,3,4),(1,2,4,3),(1,3,2,4),(1,3,4,2),(1,4,2,3),(1,4,3,2)\}$

For permutations $\pi$, where $\pi(1)=1$, we have $v\left(\bar{\tau}\left(x, \pi^{-1}(1)-1\right)\right)=1, v\left(\bar{\tau}\left(x, \pi^{-1}(1)\right)\right)=1$, $v\left(\underline{\tau}\left(x, \pi^{-1}(1)\right)\right)=0$, and $v\left(\underline{\tau}\left(x, \pi^{-1}(1)-1\right)\right)=0$, so that the value of the 4 -fold integral is given by 0 . 2.3.2. $\pi \in\{(2,1,3,4),(2,1,4,3),(3,1,2,4),(3,1,4,2),(4,1,2,3),(4,1,3,2)\}$

For permutation $\pi=(2,1,3,4)$ we consider the two cases $x_{2} \leq x_{1}$ and $x_{2} \geq x_{1}$. In both cases we have $v\left(\bar{\tau}\left(x, \pi^{-1}(1)-1\right)\right)=1$ and $v\left(\underline{\tau}\left(x, \pi^{-1}(1)-1\right)\right)=0$. For $x_{2} \leq x_{1}$ we have $v\left(\bar{\tau}\left(x, \pi^{-1}(1)\right)\right)=x_{1}$ and $v\left(\underline{\tau}\left(x, \pi^{-1}(1)\right)\right)=x_{2}$. For $x_{2} \geq x_{1}$ we have $v\left(\bar{\tau}\left(x, \pi^{-1}(1)\right)\right)=x_{2}$ and $v\left(\underline{\tau}\left(x, \pi^{-1}(1)\right)\right)=x_{1}$. Thus the corresponding 4 -fold integral is given by

$$
\begin{aligned}
& \int_{0}^{1}\left(1-\left(\int_{0}^{x_{1}} x_{1} \mathrm{~d} x_{2}+\int_{x_{1}}^{1} x_{2} \mathrm{~d} x_{2}\right)+\left(\int_{0}^{x_{1}} x_{2} \mathrm{~d} x_{2}+\int_{x_{1}}^{1} x_{1} \mathrm{~d} x_{2}\right)-0\right) \mathrm{d} x_{1} \\
& =\int_{0}^{1}\left(\frac{1}{2}+x_{1}\left(1-x_{1}\right)\right) \mathrm{d} x_{1}=\frac{2}{3}
\end{aligned}
$$

Due to symmetry we obtain the same values for all permutations $\pi$ with $\pi(2)=1$.

2.3.3. $\pi \in\{(2,3,1,4),(3,2,1,4),(2,4,1,3),(4,2,1,3),(3,4,1,2),(4,3,1,2)\}$

For permutation $\pi=(2,3,1,4)$ we consider the six strict orderings of $x_{1}, x_{2}, x_{3}$. In all six cases we have $v\left(\bar{\tau}\left(x, \pi^{-1}(1)-1\right)\right)=1$ and $v\left(\underline{\tau}\left(x, \pi^{-1}(1)-1\right)\right)=0$. If $x_{1}=\min \left(x_{2}, x_{3}\right)$ then $v\left(\bar{\tau}\left(x, \pi^{-1}(1)\right)\right)=\min \left(x_{2}, x_{3}\right)$ and $v\left(\bar{\tau}\left(x, \pi^{-1}(1)\right)\right)=x_{1}$ otherwise. If $x_{1}=\max \left(x_{2}, x_{3}\right)$ then 
$v\left(\underline{\tau}\left(x, \pi^{-1}(1)\right)\right)=\max \left(x_{2}, x_{3}\right)$ and $v\left(\underline{\tau}\left(x, \pi^{-1}(1)\right)\right)=x_{1}$ otherwise. Thus the corresponding 4-fold integral is given by

$$
\begin{aligned}
& \int_{0}^{1}\left(1-\left(\int_{x_{1}}^{1} \int_{x_{1}}^{1} \min \left(x_{2}, x_{3}\right) \mathrm{d} x_{2} \mathrm{~d} x_{3}+\int_{0}^{x_{1}} \int_{x_{3}}^{1} x_{1} \mathrm{~d} x_{2} \mathrm{~d} x_{3}+\int_{0}^{x_{1}} \int_{x_{2}}^{1} x_{1} \mathrm{~d} x_{3} \mathrm{~d} x_{2}\right)\right. \\
& \left.+\left(\int_{0}^{x_{1}} \int_{0}^{x_{1}} \max \left(x_{2}, x_{3}\right) \mathrm{d} x_{2} \mathrm{~d} x_{3}+\int_{x_{1}}^{1} \int_{0}^{x_{3}} x_{1} \mathrm{~d} x_{2} \mathrm{~d} x_{3}+\int_{x_{1}}^{1} \int_{0}^{x_{2}} x_{1} \mathrm{~d} x_{3} \mathrm{~d} x_{2}\right)-0\right) \mathrm{d} x_{1} \\
& =\int_{0}^{1}\left(\frac{2}{3}-x_{1}^{2}+x_{1}\right) \mathrm{d} x_{1}=\frac{5}{6}
\end{aligned}
$$

Due to symmetry we obtain the same values for all permutations $\pi$ with $\pi(3)=1$.

2.3.4. $\pi \in\{(2,3,4,1),(3,2,4,1),(2,4,3,1),(4,2,3,1),(3,4,2,1),(4,3,2,1)\}$

For permutation $\pi=(2,3,4,1)$ we have $v\left(\bar{\tau}\left(x, \pi^{-1}(1)\right)\right)=v\left(\underline{\tau}\left(x, \pi^{-1}(1)\right)\right), v\left(\bar{\tau}\left(x, \pi^{-1}(1)-1\right)\right)=$ $\max \left(x_{2}, x_{3}, x_{4}\right)$, and $v\left(\underline{\tau}\left(x, \pi^{-1}(1)-1\right)\right)=\min \left(x_{2}, x_{3}, x_{4}\right)$. Thus the corresponding 4-fold integral is given by

$$
\int_{0}^{1}\left(\int_{x=\left(x_{2}, x_{3}, x_{4}\right) \in[0,1]^{3}}\left(\max \left(x_{2}, x_{3}, x_{4}\right)-\min \left(x_{2}, x_{3}, x_{4}\right)\right) \mathrm{d} x\right) \mathrm{d} x_{1}=\int_{0}^{1} \frac{\mathrm{d} x_{1}}{2}=\frac{1}{2}
$$

Due to symmetry we obtain the same values for all permutations $\pi$ with $\pi(4)=1$.

2.3.5. Summarizing the 24 Permutations for $\operatorname{SSI}_{1}(v)$

$$
\operatorname{SSI}_{1}(v)=\frac{1}{4 !} \cdot\left(6 \cdot 0+6 \cdot \frac{2}{3}+6 \cdot \frac{5}{6}+6 \cdot \frac{1}{2}\right)=\frac{12}{24}=\frac{1}{2}
$$

\section{Determining the Nuc for Two Continuous Simple Games}

From the binary case we can learn that very often it suffices to just minimize the maximum excess in order to compute the nucleolus of a simple game. So for example $\hat{v}$ we maximizing the excess

$$
\frac{x_{1}^{2}+2 x_{2}^{2}+3 x_{3}^{2}}{6}-w_{1} x_{1}-w_{2} x_{2}-\left(1-w_{1}-w_{2}\right) x_{3}
$$

subject to the constraints $0 \leq x_{1}, x_{2}, x_{3} \leq 1$. With the aid of the corresponding Lagrange function $L\left(x_{1}, x_{2}, x_{3}, \alpha_{1}, \alpha_{2}, \alpha_{3}, \beta_{1}, \beta_{2}, \beta_{3}\right)=$

$$
\begin{aligned}
& \frac{x_{1}^{2}+2 x_{2}^{2}+3 x_{3}^{2}}{6}-w_{1} x_{1}-w_{2} x_{2}-\left(1-w_{1}-w_{2}\right) x_{3}+\alpha_{1} x_{1} \\
& +\alpha_{2} x_{2}+\alpha_{3} x_{3}-\beta_{1}\left(x_{1}-1\right)-\beta_{2}\left(x_{2}-1\right)-\beta_{3}\left(x_{3}-1\right)
\end{aligned}
$$


we conclude that the maximum excess is attained at one of the solutions of the following equation system:

$$
\begin{aligned}
\frac{\partial L}{\partial x_{1}}\left(x_{1}, \ldots, \beta_{3}\right)=\frac{x_{1}}{3}-w_{1}+\alpha_{1}-\beta_{1} & \stackrel{!}{=} 0 \\
\frac{\partial L}{\partial x_{2}}\left(x_{1}, \ldots, \beta_{3}\right)=\frac{2 x_{2}}{3}-w_{2}+\alpha_{2}-\beta_{2} & \stackrel{!}{=} 0 \\
\frac{\partial L}{\partial x_{3}}\left(x_{1}, \ldots, \beta_{3}\right)=x_{3}-w_{3}+\alpha_{3}-\beta_{3} & \stackrel{!}{=} 0 \\
\alpha_{1} x_{1} & \stackrel{!}{=} 0 \\
\alpha_{2} x_{2} & \stackrel{!}{=} 0 \\
\alpha_{3} x_{3} & \stackrel{!}{=} 0 \\
\beta_{1}\left(x_{1}-1\right) & \stackrel{!}{=} 0 \\
\beta_{2}\left(x_{2}-1\right) & \stackrel{!}{=} 0 \\
\beta_{3}\left(x_{3}-1\right) & \stackrel{!}{=} 0
\end{aligned}
$$

where we have set $w_{3}=1-w_{1}-w_{2}$. The resulting 27 solutions $\left(x_{1}, x_{2}, x_{3}\right)$ are given by

$$
\begin{array}{rllll}
x_{1}=3 w_{1} & \vee & x_{1}=0 & \vee & x_{1}=1 \\
x_{2}=\frac{3 w_{2}}{2} & \vee & x_{2}=0 & \vee & x_{2}=1 \\
x_{3}=w_{3} & \vee & x_{3}=0 & \vee & x_{3}=1
\end{array}
$$

For each of these 27 combinations we can compute the excess $e^{v}(x, w)$, where $x$ is specified by $w$. Now we are just looking at the 8 solutions where $x \in\{0,1\}^{3}$ and compute the respective excesses:

$$
\begin{aligned}
e^{v}((0,0,0), w) & =0, \\
e^{v}((1,0,0), w) & =\frac{1}{6}-w_{1}, \\
e^{v}((0,1,0), w) & =\frac{1}{3}-w_{2}, \\
e^{v}((0,0,1), w) & =\frac{1}{2}-w_{3}, \\
e^{v}((1,1,0), w) & =w_{3}-\frac{1}{2}, \\
e^{v}((1,0,1), w) & =w_{2}-\frac{1}{1}, \\
e^{v}((0,1,1), w) & =w_{1}-\frac{1}{6}, \\
e^{v}((1,1,1), w) & =0
\end{aligned}
$$

Thus we have that the maximum excess is at least

$$
\max \left(\left|\frac{1}{6}-w_{1}\right|,\left|\frac{1}{3}-w_{2}\right|,\left|\frac{1}{2}-w_{3}\right|\right) \geq 0
$$

More precisely, each choice of $w \neq \frac{1}{6} \cdot(1,2,3)$ leads to a maximum excess larger than 0 . Next we show that choosing $w=\frac{1}{6} \cdot(1,2,3)$ yields a maximum excess of 0 , so that $\operatorname{Nuc}(v)=\left\{\frac{1}{6} \cdot(1,2,3)\right\}$. It 
remains to prove $e^{v}\left(x, \frac{1}{6} \cdot(1,2,3)\right) \leq 0$ for all $x \in[0,1]^{3}$. We may check all 27 solutions and verify that the excess at these points is at most 0 or simply rewrite the formula for the maximum excess at $w=\frac{1}{6} \cdot(1,2,3)$ to

$$
e^{v}\left(x, \frac{1}{6} \cdot(1,2,3)\right)=-\frac{x_{1}\left(1-x_{1}\right)}{6}-\frac{x_{2}\left(1-x_{2}\right)}{3}-\frac{x_{3}\left(1-x_{3}\right)}{2}
$$

which is obviously upper bounded by zero for all $x \in[0,1]^{3}$.

For our second example minimizing the maximum excess does not help very much. Using the fact that the geometric mean is always at most as large as the arithmetic mean we deduce

$$
x_{1} x_{2}^{2} x_{3}^{3} \leq \sqrt[n]{x_{1} x_{2}^{2} x_{3}^{3}} \leq \frac{x_{1}+2 x_{2}+3 x_{3}}{n}
$$

for all integers $n$. If $w_{1}, w_{2}, w_{3}>0$ the right hand side is at most $w_{1} x_{1}+w_{2} x_{2}+w_{3} x_{3}$ for suitably large $n$. Using an easy but elaborated argument for the cases where some of the $w_{i}$ but not all are zero, we can conclude $e^{v}(x, w) \leq 0$ for all $x \in[0,1]^{3}$ and all $w \in[0,1]^{3}$ satisfying $\|w\|_{1}=1$. Thus from the maximum excess we can only conclude the trivial implication $\operatorname{Nuc}(v) \in\left\{w \in[0,1]^{3}:\|w\|_{1}\right\}$.

In order to get an idea of how the nucleolus may be computed in similar cases, we have considered the more simple two voter example $v\left(x_{1}, x_{2}\right)=x_{1} x_{2}^{2}$. Here minimizing the maximum excess is of little use too. A feasible approach might be to compute an exact expression for the excess function in the domain $[c, 1]$, where $c<1$ is near to one, parametric in $w_{1}$ and $w_{2}=1-w_{1}$. Using a somewhat elaborated case distinction and assuming a $c$ sufficiently close to 1 , we were able to state an exact formula for the excess function using a sum of several integrals. Those integrals could be evaluated numerically for fix values of $w_{1}, w_{2}$. By plotting and comparing the excess functions for certain weights, we were able to compute the numerical bounds $0.4553 \leq y_{1} \leq 0.4555$ and $0.5545 \leq y_{2} \leq 0.5547$ for all $\left(y_{1}, y_{2}\right) \in \operatorname{Nuc}(v)$.

(c) 2014 by the author; licensee MDPI, Basel, Switzerland. This article is an open access article distributed under the terms and conditions of the Creative Commons Attribution license (http://creativecommons.org/licenses/by/3.0/). 